\title{
A COMPLETE CHARACTERIZATION OF INVARIANT JOINTLY RANK- $R$ CONVEX QUADRATIC FORMS AND APPLICATIONS TO COMPOSITE MATERIALS
}

\author{
VINCENZO NESI ${ }^{1}$ AND ENRICO ROGORA ${ }^{1}$
}

\begin{abstract}
The theory of compensated compactness of Murat and Tartar links the algebraic condition of rank- $r$ convexity with the analytic condition of weak lower semicontinuity. The former is an algebraic condition and therefore it is, in principle, very easy to use. However, in applications of this theory, the need for an efficient classification of rank- $r$ convex forms arises. In the present paper, we define the concept of extremal 2 -forms and characterize them in the rotationally invariant jointly rank- $r$ convex case.
\end{abstract}

Mathematics Subject Classification. 74Q20, 49K20, 35J50, 74E30.

Received October 27, 2004. Revised June 30, 2005.

\section{INTRODUCTION}

The theory of compensated compactness $[42,59]$ links the algebraic condition of rank- $r$ convexity with the analytic condition of weak lower semicontinuity. To make this statement precise we need to introduce some definitions. Throughout the paper the integer $d \geq 2$ will denote the dimension of the vector space $\mathbb{R}^{d}$ over which the relevant fields will be defined. We denote the space of real $n \times m$ matrices by

$$
\mathcal{M}(n, m)
$$

and we set

$$
\mathcal{M}^{k}(n, m):=\overbrace{\mathcal{M}(n, m) \times \cdots \times \mathcal{M}(n, m)}^{k \text { times }} .
$$

Definition 1.1. We use a bold character, like $\mathbf{P}$, to denote a list of matrices $\left(P^{1}, P^{2}, \ldots, P^{k}\right)$. We denote by $O(d)$ the group of real orthogonal matrices i.e. the matrices $M \in \mathcal{M}(d, d)$ such that $M M^{t}=\operatorname{Id}(d)$.

Definition 1.2. A function

$$
F: \mathcal{M}^{k}(d, d) \rightarrow \mathbb{R}
$$

is a 2 -form if it is a homogeneous polynomial of degree two.

Keywords and phrases. Compensated compactness, rank- $r$ convexity, effective conductivity, quadratic forms.

1 Dipartimento di Matematica, Università di Roma "La Sapienza", Italy; nesi@mat.uniroma1.it; rogora@mat.uniroma1.it

(c) EDP Sciences, SMAI 2007 
Definition 1.3. Let $r$ be an integer with $r \in\{1, \ldots, d\}$. A function $F$ is an invariant 2-form if it is a 2 -form which is rotationally invariant in the following sense

$$
\begin{gathered}
\forall R \in O(d), \quad \forall \mathbf{M}=\left(M^{1}, M^{2}, \ldots, M^{k}\right) \in \mathcal{M}^{k}(d, d), \\
F\left(M^{1}, M^{2}, \ldots, M^{k}\right)=F\left(R^{t} M^{1} R, R^{t} M^{2} R, \ldots, R^{t} M^{k} R\right) .
\end{gathered}
$$

Definition 1.4. A function $F: \mathcal{M}(d, d) \rightarrow \mathbb{R}$ is rank-r convex if

$$
F((1-t) P+t Q) \leq(1-t) F(P)+t F(Q)
$$

for all $t \in[0,1]$ and all pair of matrices $P$ and $Q$ in $\mathcal{M}(d, d)$ such that $\operatorname{rank}(Q-P) \leq r$ or, equivalently, such that $\operatorname{dim}(\operatorname{Ker}(Q-P)) \geq d-r$.

Remark 1.5. The notion of $d$-convexity is equivalent to the usual notion of convexity.

Remark 1.6. A 2 -form $F$ is rank- $r$ convex if and only if

$$
F(P) \geq 0, \quad \forall P \in \mathcal{M}(d, d) \quad \text { such that } \quad \operatorname{dim}(\operatorname{Ker}(P)) \geq d-r .
$$

In particular, $F$ is convex (equivalently, rank- $d$ convex) if and only if

$$
F(P) \geq 0, \quad \forall P \in \mathcal{M}(d, d) .
$$

Notation. Let $\Omega$ be an open bounded subset of $\mathbb{R}^{d}$ and let $p \in L^{2}\left(\Omega ; \mathbb{R}^{d}\right), p=\left(p_{1}, \ldots, p_{d}\right)$. We define Curl $p \in H^{-1}\left(\Omega ; \mathbb{R}^{d \times d}\right)$ by

and Div $p \in H^{-1}(\Omega ; \mathbb{R})$ by

$$
(\operatorname{Curl} p)_{i j}=\frac{\partial p_{i}}{\partial x_{j}}-\frac{\partial p_{j}}{\partial x_{i}}, \quad i, j=1, \ldots, d
$$

$$
\text { Div } p=\frac{\partial p_{1}}{\partial x_{1}}+\cdots+\frac{\partial p_{d}}{\partial x_{d}}
$$

The definitions of Div and Curl carry over to matrix valued functions by defining Div and Curl row by row. Namely, if $P \in L^{2}\left(\Omega ; \mathbb{R}^{d \times d}\right)$ and $p^{k}$ is the $k$-th row of $P$, then Curl $P \in H^{-1}\left(\Omega ; \mathbb{R}^{d \times d \times d}\right)$ is defined by

$$
(\operatorname{Curl} P)_{i j k}=\frac{\partial p_{i}^{k}}{\partial x_{j}}-\frac{\partial p_{j}^{k}}{\partial x_{i}}, \quad i, j, k=1, \ldots, d,
$$

and Div $P \in H^{-1}\left(\Omega ; \mathbb{R}^{d}\right)$ is defined by

$$
(\operatorname{Div} P)_{k}=\frac{\partial p_{1}^{k}}{\partial x_{1}}+\cdots+\frac{\partial p_{d}^{k}}{\partial x_{d}} \quad k=1, \ldots, d .
$$

The following two remarkable results are well known and establish the importance of the notion of $r$-convexity.

Theorem 1.7 (Tartar [58]). Let $\Omega$ be an open bounded subset of $\mathbb{R}^{d}$ and let $P_{\epsilon}$ and $P_{0}$ be in $L^{2}\left(\Omega ; \mathbb{R}^{d \times d}\right)$ such that

$$
P_{\epsilon} \rightarrow P_{0} \text { weakly in }\left(L^{2}(\Omega)\right)^{d \times d}
$$

Curl $P_{\epsilon}$ belongs to a compact subset of $\left(H^{-1}(\Omega)\right)^{d \times d \times d}$.

Let $F$ be a rank-1 convex 2 -form on $\mathcal{M}(d, d)$. Then, for any $\phi \geq 0$ with $\phi \in C_{c}(\Omega)$, we have

$$
\liminf _{\epsilon \rightarrow 0^{+}} \int_{\Omega} \phi F\left(P_{\epsilon}\right) \mathrm{d} x \geq \int_{\Omega} \phi F\left(P_{0}\right) \mathrm{d} x .
$$


Theorem 1.8 (Tartar [58]). Let $\Omega$ be an open bounded subset of $\mathbb{R}^{d}$ and let $Q_{\epsilon}$ and $Q_{0}$ be in $L^{2}\left(\Omega ; \mathbb{R}^{d \times d}\right)$ such that

$$
Q_{\epsilon} \rightarrow Q_{0} \text { weakly in }\left(L^{2}(\Omega)\right)^{d \times d},
$$

$\operatorname{Div} Q_{\epsilon}$ belongs to a compact subset of $\left(H^{-1}(\Omega)\right)^{d}$.

Let $F$ be a rank- $(d-1)$ convex 2 -form on $\mathcal{M}(d, d)$. Then, for any $\phi \geq 0$ with $\phi \in C_{c}(\Omega)$, we have

$$
\liminf _{\epsilon \rightarrow 0^{+}} \int_{\Omega} \phi F\left(Q_{\epsilon}\right) \mathrm{d} x \geq \int_{\Omega} \phi F\left(Q_{0}\right) \mathrm{d} x
$$

In his work on bounds in homogenization [57], [58] Tartar observed that the function

$$
T^{(1)}(E):=\operatorname{Tr}\left(E E^{t}\right)-(\operatorname{Tr} E)^{2}
$$

is rank-1 convex, whereas the function

$$
T^{(d-1)}(B):=(d-1) \operatorname{Tr}\left(B B^{t}\right)-(\operatorname{Tr} B)^{2}
$$

is rank- $(d-1)$ convex. Hence, Theorem 1.7 holds with $F=T^{(1)}$ and Theorem 1.8 holds with $F=T^{(d-1)}$. See [61] for a detailed derivation of bounds in homogenization using these ideas.

The main theme of the present paper is to describe the set of all invariant, jointly (in the sense of Def. 1.9), rank- $r$ convex 2-forms in a way which makes their use in homogenization most efficient. Before explaining our contribution toward this goal, let us mention why the natural setting is with a 2 -form defined on $\mathcal{M}^{k}(d, d)$, for possibly very large $k$. Suppose we try to bound the effective conductivity tensor (also called $H$-limit [45] or $G$-limit [53]) of some family of conductivity matrices $\sigma_{\epsilon}$ defined on some subdomain of $\mathbb{R}^{d}$. The natural variables are an "almost irrotational" sequence of fields $e_{\epsilon}(x)$ (in the sense of (1.8)) and an "almost soloneoidal" sequence of fields $b_{\epsilon}(x)$ (in the sense of $(1.11)$ ). It turns out that it is useful to consider $d$ different choices of pairs $\left(b_{\epsilon}, e_{\epsilon}\right)$ which, in some sense, are relative to linearly independent boundary conditions (see Section 5 for more details in the periodic context). Therefore the basic sequences can be thought of as a $d \times d$ almost irrotational matrix valued field $E_{\epsilon}$ and a $d \times d$ almost solenoidal matrix valued field $B_{\epsilon}$ related by the linear equations $\sigma_{\epsilon} E_{\epsilon}^{t}=B_{\epsilon}^{t}$. This case is covered setting $k=1$.

A different and interesting problem is the study of the effective properties of $k$ distinct physical properties $[4,5,10,12,13,19,22-24,33-35,52,58]$. This is one instance where the need for $k>1$ arises. Another example is the case when measurements for the effective properties are known for certain values of the conductivity of the constituent phases. One would like to have bounds for the effective properties for different values of the conductivity of the constituent phases. Work in this direction includes $[7,8,35,36,49]$.

More recently, yet another field of possible applications arose from the work on bounding effective laws or overall energies for non linear (for instance power law) composites. The pertinent number $k$ in this framework turns out to be unbounded! See $[25,40]$. We will give further simpler motivations in Section 6 .

For these kind of applications (and many others) we need to introduce the definition which makes compensated compactness applicable in a straightforward way.

Definition 1.9. Let $r$ and $k$ be integers with $1 \leq r \leq d$ and $1 \leq k$. We say that $F$ is jointly rank-r convex if $F((1-t) \mathbf{P}+t \mathbf{Q}) \leq(1-t) F(\mathbf{P})+t F(\mathbf{Q})$ for all $t \in[0,1]$ and all pairs of choices $\mathbf{P}=\left(P^{1}, P^{2}, \ldots, P^{k}\right)$, $\mathbf{Q}=\left(Q^{1}, Q^{2}, \ldots, Q^{k}\right) \in \mathcal{M}^{k}(d, d)$ such that

$$
\operatorname{dim}\left(\bigcap_{i=1}^{k} \operatorname{Ker}\left(Q^{i}-P^{i}\right)\right) \geq d-r .
$$

We will often omit the qualifier "jointly" in the rest of the paper. 
Remark 1.10. When $F$ is a 2 -form, the Definition 1.9 reduces itself to the following condition: $F$ is jointly rank- $r$ convex if and only if

$$
F(\mathbf{P}) \geq 0, \quad \forall \mathbf{P} \in \mathcal{M}^{k}(d, d) \quad \text { such that } \operatorname{dim}\left(\bigcap_{i=1}^{k} \operatorname{Ker}\left(P^{i}\right)\right) \geq d-r .
$$

Remark 1.11. Let us point out some very elementary facts about joint rank- $r$ convexity of 2 -forms. As already mentioned, rank- $d$ convexity is just the usual convexity and rank- $r$ convexity implies rank- $(r-1)$ convexity for all $r \in\{d, d-1, \ldots, 2\}$. The sum of a rank- $r_{1}$ convex form and a rank- $r_{2}$ convex form is rank- $r$ convex with $r=\min \left(r_{1}, r_{2}\right)$. In particular the sum of a rank- $r$ convex and a convex form, is rank- $r$ convex.

Let us now recall that convex forms are not interesting in the framework of compensated compactness because they carry no differential information. To be specific, assume that $F$ is rank-1 convex and $F=\mathcal{C}+\mathcal{Q}$ with $\mathcal{C}$ convex and $\mathcal{Q}$ rank-1 convex. By rank-1 convexity of $F$ and $\mathcal{Q}$ one has: for all sequences $\left\{E_{\epsilon}\right\}$ satisfying $(1.7)$ and (1.8)

$$
\begin{gathered}
\liminf _{\epsilon \rightarrow 0^{+}}\left(\int_{\Omega} \phi \mathcal{C}\left(E_{\epsilon}\right)+\int_{\Omega} \phi \mathcal{Q}\left(E_{\epsilon}\right)\right) \geq \int_{\Omega} \phi \mathcal{C}\left(E_{0}\right)+\int_{\Omega} \phi \mathcal{Q}\left(E_{0}\right) \quad \forall \phi \in C_{c}(\Omega), \phi \geq 0, \\
\liminf _{\epsilon \rightarrow 0^{+}} \int_{\Omega} \phi \mathcal{Q}\left(E_{\epsilon}\right) \geq \int_{\Omega} \phi \mathcal{Q}\left(E_{0}\right) \forall \phi \in C_{c}(\Omega), \phi \geq 0 .
\end{gathered}
$$

By the convexity of $\mathcal{C}$ one has: for all sequences $\left\{A_{\epsilon}\right\}$ satisfying (1.7) (but not necessarily (1.8)!)

$$
\liminf _{\epsilon \rightarrow 0^{+}} \int_{\Omega} \phi \mathcal{C}\left(A_{\epsilon}\right) \geq \int_{\Omega} \phi \mathcal{C}\left(A_{0}\right)
$$

Clearly (1.17) and (1.18) imply (1.16) but only (1.17) is a consequence of the differential constraint (1.8). In contrast (1.18) does not carry the information (1.8).

This observation has a counterpart in the issue of bounding effective moduli. In fact, as discovered by Milton [36] and Cherkaev and Gibiansky [20], in order to bound effective moduli of composite materials, the "useful" rank-1 convex functions are those which cannot be decomposed as a sum of a possibly different rank1 convex function and a convex one. Similarly for the case in which one uses a rank- $(d-1)$ convex function. The same issue appears in the context of linearized elasticity. A striking example can be found in the work of Allaire and Kohn [2] where the authors implements the ideas of Milton [36] in the specific example of seeking lower bounds on the elastic energy of a $2 D$ composite. In Section 5 we will give more details explaining what is meant by "useful" rank- $r$ convex 2-forms. The key observation can be found in Lemma 5.5. Roughly speaking the lemma states that the bounds obtained by using a class of 2 -forms $F=\mathcal{C}+\mathcal{Q}$ with $\mathcal{C}$ ranging on a set of convex 2 -forms and $\mathcal{Q}$ a given rank-1 convex 2 -form, do not improve upon those using only the function $\mathcal{Q}$. We are led to the following definition which is the essential basis of our work.

Definition 1.12. Let $r, s, k$ be integers such that $1 \leq r<s \leq d$ and $1 \leq k$ and let $F$ be a non zero rank- $r$ convex invariant 2 -form on $\mathcal{M}^{k}(d, d)$. We say that $F$ is quadratically $(r, s)$ extremal if the following condition holds: assume $F=F_{r}+F_{s}$ with $F_{r}$ a rank- $r$ convex invariant 2-form and $F_{s}$ a rank- $s$ convex invariant 2-form, then $F_{s}$ is identically zero.

We will typically omit the qualifier "quadratically".

Our definition of extremal forms can be rephrased as follows: for $r$ and $s$ as in Definition 1.12, we say that the rank- $r$ convex form $F$ is $(r, s)$ extremal if one cannot subtract from $F$ any rank- $s$ convex form $(s>r)$ without 
loosing rank- $r$ convexity of $F$. Note that if $F$ is $(r, s)$ extremal and $s<d$ then $F$ is also $(r, s+1)$ extremal. For the converse statement, see Proposition 1.20.

The nature of our results suggests an independent definition for the case $r=s$.

Definition 1.13. Let $r$ and $k$ be integers such that $1 \leq r \leq d$ and $1 \leq k$ and let $F$ be a rank- $r$ convex invariant 2 -form on $\mathcal{M}^{k}(d, d)$. We say that $F$ is quadratically $(r, r)$ extremal if it cannot be expressed as the sum of two linearly independent invariant rank- $r$ convex 2 -forms.

Our main result is to give a complete characterization of $(r, s)$ extremal invariant 2-forms for all integers $r, s$ with $1 \leq r \leq d$ and $r \leq s \leq d$ (see Prop. 1.19). At present, applications to composites are restricted to the case $r=1$ and $r=d-1$ but we believe that the general theory will turn out to be equally useful. Let us now describe a typical result of our analysis. It deals with the familiar case of rank- $(d-1)$ convexity and therefore with the case of almost divergence free matrix fields.

Proposition 1.14. Let $d \geq 3$ and $k \geq 1$ be two given integers. Let $F$ be a jointly rank-(d-1) convex invariant 2 -form. Then $F$ is quadratically $(d-1, d)$ extremal if and only if there exists a symmetric, nonnegative matrix $H$ with elements $h_{i j}$ such that

$$
F(\mathbf{M})=\sum_{i, j=1}^{k} h_{i j} \mathcal{G}\left(M^{i}, M^{j}\right)
$$

where

$$
\mathcal{G}(P, Q):=\frac{d-1}{2}\left[\operatorname{Tr}\left(P Q^{t}\right)+\operatorname{Tr}(P Q)\right]-\operatorname{Tr}(P) \operatorname{Tr}(Q) .
$$

Furthermore $F$ is $(d-1, d-1)$ extremal if and only if it has the form (1.19), for some symmetric matrix $H \geq 0$, with $\operatorname{rank}(H)=1$. Finally any rank- $(d-1)$ convex invariant 2 -form $F$ is the sum of a convex one and a $(d-1, d)$ extremal one.

Proposition 1.14 follows from Theorem 1.15 and Proposition 1.19, which treat the more general case $r \in$ $\{2, \ldots, d\}$. We now state our results in their general form.

Theorem 1.15. Let $d \geq 3$ and $1 \leq k$ be two integers. Let $r$ and $s$ be two integers with $2 \leq r<s \leq d$. Let $F$ be a non zero, jointly rank-r convex, invariant 2-form. Then,

Part I) $F$ is quadratically $(r, s)$ extremal if and only if there exists a symmetric matrix $H \geq 0$ with elements $h_{i j}$ such that

$$
F(\mathbf{M})=\sum_{i, j=1}^{k} h_{i j} \mathcal{G}_{r}\left(M^{i}, M^{j}\right)
$$

where

$$
\mathcal{G}_{r}(P, Q):=\frac{r}{2}\left[\operatorname{Tr}\left(P Q^{t}\right)+\operatorname{Tr}(P Q)\right]-\operatorname{Tr}(P) \operatorname{Tr}(Q) .
$$

Part II) Furthermore, $F$ is, in addition, quadratically $(r, r)$ extremal if and only if it has the form (1.21) for some symmetric matrix $H \geq 0$ and with $\operatorname{rank}(H)=1$.

The case $r=1$ is handled by the following result.

Theorem 1.16. Let $d \geq 2$ and $k \geq 1$ be two integers. Let $s$ be an integer with $1<s \leq d$. Let $F$ be a non zero, jointly rank-1 convex, invariant 2 -form. Then,

Part I) $F$ is quadratically $(1, s)$ extremal if and only if there exists an arbitrary symmetric matrix $H$ with elements $h_{i j}$ such that

$$
F(\mathbf{M})=\sum_{i, j=1}^{k} h_{i j} \mathcal{G}_{1}\left(M^{i}, M^{j}\right)
$$


where

$$
\mathcal{G}_{1}(P, Q):=\operatorname{Tr}(P Q)-\operatorname{Tr}(P) \operatorname{Tr}(Q) .
$$

Part II) Furthermore, $F$ is, in addition, quadratically $(1,1)$ extremal if and only if it has the form (1.23) for some $H$ with $\operatorname{rank}(H)=1$.

Remark 1.17. When $r=1$, the function $E \rightarrow \mathcal{G}_{1}(E, E)$ is well known. It is the (unique up to a multiplicative factor) quadratic invariant null-lagrangian. When $r=d-1$, the importance of the function $B \rightarrow \mathcal{G}_{d-1}(B, B)$ has been already recognized by Cherkaev and Gibiansky [20] and by Milton [36].

To simplify the following statements it is convenient to have a single notation for (1.21) and (1.23).

Definition 1.18. Given an integer $k \geq 1$, an integer $r \in\{1,2, \ldots, d-1\}$ and a symmetric $k \times k$ matrix $H$ (nonnegative definite if $r \geq 2$ ), we define the following forms $\mathcal{F}_{H}^{(r)}: \mathcal{M}^{k}(d, d) \rightarrow \mathbb{R}$

$$
\mathcal{F}_{H}^{(r)}(\mathbf{M}):=\sum_{i, j=1}^{k} h_{i j} \mathcal{G}_{r}\left(M^{i}, M^{j}\right)
$$

where the forms $\mathcal{G}_{r}$ are defined in (1.22) for $r \geq 2$ and in (1.24) for $r=1$.

In the language of Definitions 1.13 and 1.18, the jointly rank- $r$ convex, $(r, r+1)$ extremal forms are exactly the forms $\mathcal{F}_{H}^{(r)}$ defined in (1.25). We can now state two useful corollaries of our results.

Proposition 1.19. (Canonical form of jointly rank- $r$ convex invariant 2-forms). Let $k$ and $r$ be two integers, $1 \leq k, 1 \leq r \leq d$. Any invariant, jointly rank-r convex 2 -form $F$ on $\mathcal{M}^{k}(d, d)$ is the sum of at most $k$ invariant and $(r, r)$-extremal, 2-forms and of an invariant and convex one. More precisely, there exist at most $k$ linearly independent vectors $u^{j} \in \mathbb{R}^{k}$ and a convex form $\mathcal{C}$ such that

$$
F(\mathbf{M})=\left\{\begin{array}{cc}
\sum_{j=1}^{k} \mathcal{F}_{u^{j} \otimes u^{j}}^{(r)}(\mathbf{M})+\mathcal{C}(\mathbf{M}) & \text { if } r \geq 2 \\
\sum_{j=1}^{k} \sigma_{j} \mathcal{F}_{u^{j} \otimes u^{j}}^{(r)}(\mathbf{M})+\mathcal{C}(\mathbf{M}) & \text { if } r=1
\end{array}\right.
$$

with $\sigma_{j} \in\{-1,1\}$ for $j=1, \ldots, k$.

Proposition 1.20. Let $F$ be a jointly rank-r convex, invariant 2-form on $\mathcal{M}^{k}(d, d)$. Then $F$ is $(r, d)$-extremal if and only if it is $(r, r+1)$-extremal.

Remark 1.21. It is easy to verify that the form (1.26) can be equivalently rewritten as follows:

$$
F(\mathbf{M})=\sum_{j=1}^{k} G^{(r)}\left(\sum_{i=1}^{k} M^{i} u_{i}^{j}\right)+\mathcal{C}(\mathbf{M})
$$

where

Hence, defining $P_{j}:=\sum_{i=1}^{k} M^{i} u_{i}^{j}$, one has

$$
G^{(r)}(P):=\mathcal{G}_{r}(P, P)
$$

$$
F(\mathbf{M})=\sum_{j=1}^{k} G^{(r)}\left(P_{j}\right)+\mathcal{C}(\mathbf{M})
$$


The latter formula shows that, for given $r$, essentially only one form has to be remembered, namely $G^{(r)}$ !

Remark 1.22. The decomposition (1.26) is not unique as pointed out by one of the referees. However, given $F$, we will give an explicit algorithm to construct one possible choice of the vectors $u^{j}$, the form $\mathcal{C}$ and the numbers $\sigma_{j}$. We will came back to this issue in Remark 3.4.

The plan of the paper is the following. In Section 2 we review some well known facts from group representation theory and collect a few facts of linear algebra which will be used in Section 3. In Section 3 we use the results of Section 2 to prove Lemma 3.1, which is the main tool used to prove Theorems 1.15 and 1.16 and Propositions 1.19 and 1.20. Lemma 3.1 gives a necessary and sufficient condition to determine when $F$ is jointly rank- $r$ convex. In Section 4, we present more detailed results concerning extremal rank- $r$ functions aiming, in particular, at characterizing the sets where the extremal forms are positive, null and negative respectively. When $k=1$ and $r=d-1$, our results recover those of Milton [38], p. 546. We also present a characterization of extremal forms (see Prop. 4.3). These results are essential to establish necessary conditions on the relevant fields for optimality of bounds using the compensated compactness method.

In Section 5, we present an interesting application of our results to composites. We focus on the effective properties of $3 D$ materials made of two distinct phases each of which has two distinctive physical properties such as electrical conductivity and magnetic permeability. Application of the theory developed in Sections 2 and 3, delivers bounds which were found in [5] (lower bounds) and in [7]; see also [6,34,35] (upper bounds). The novelty is two-fold. First the upper bounds were not found before using the compensated compactness method (in contrast, for the case $d=2$, Cherkaev and Gibiansky [12], have a complete characterization based on compensated compactness). Second, and most important, our work shows that the bounds cannot be improved by a more clever choice of the invariant rank- $(d-1)$ forms in the class of those which are rotationally invariant. At present the upper bound is only known to be optimal at five specific points. Therefore one should either consider 2-forms which are not rotationally invariant or change the bounding method to include more detailed information about the specific problem under consideration, like in [48]. A third possibility is to look for new microgeometries. In fact, non invariant 2-forms have already been considered by Dell'Antonio and Nesi [16] in the context of linear conductivity and by Gibiansky and Cherkaev in the context of linear elasticity [20,21]. We remark that although we refrain ourselves to present the details, our approach permits to treat anisotropic composites, a task which seems beyond the capabilities of the method based on analytic dependency exploited in $[34,35]$. Finally, in Section 6 , we generalize our approach in such a way to be able to cover the setting of the div-curl lemma of Tartar and Murat $[42,59]$. Indeed we consider forms acting simultaneously on fields of "almost gradient" type and of "almost divergence" type. Our results are not conclusive for these cases because we have not achieved a complete description of "extremal forms". However we prove an algebraic lemma, Lemma 6.7, that reduces the calculation to a set of inequalities involving $k \times k$ matrices for any given $k$. Moreover, in Corollary 6.9 we characterize, for any $k$, those forms for which the results which are the analogous of Theorems 1.7 and 1.8 hold both for $F$ and $-F$, effectively characterizing what should be called "null lagrangians" in this context. Overall our results should be seen as a generalization of the work of Milton and Cherkaev and Gibiansky attempting to make optimal use of the compensated compactness statements. Many open questions remain.

\section{TOOLS FROM REPRESENTATION THEORY}

Let $\mathcal{M}(d, d)$ be the algebra of $d \times d$ matrices with real coefficient, endowed with the inner product

$$
\langle A, B\rangle:=\operatorname{Tr}\left(A B^{t}\right) .
$$

Recall that the orthogonal group $O(d)$ is the group of matrices $R \in \mathcal{M}(d, d)$ such that $R R^{t}=I d(d)$ acts on $\mathcal{M}(d, d)$ by

$$
\begin{array}{cl}
O(d) \times \mathcal{M}(d, d) & \longrightarrow \mathcal{M}(d, d) \\
(R, M) & \longmapsto \\
R M R^{t} .
\end{array}
$$


This induces the action

$$
\begin{array}{ccc}
O(d) \times \mathcal{M}^{k}(d, d) & \longrightarrow & \mathcal{M}^{k}(d, d) \\
(R, \mathbf{M})=\left(R,\left(M_{1}, \ldots, M_{k}\right)\right) & \longmapsto & \left(R M_{1} R^{t}, \ldots, R M_{k} R^{t}\right)=R \mathbf{M} R^{t},
\end{array}
$$

and the contragradient action

$$
\begin{array}{ccc}
O(d) \times \mathbb{R}\left[x_{i j}^{1}, \ldots, x_{i j}^{k}\right] & \longrightarrow & \mathbb{R}\left[x_{i j}^{1}, \ldots, x_{i j}^{k}\right] \\
\left(R, f\left(X_{1}, \ldots, X_{k}\right)\right) & \longmapsto & f\left(R^{t} X_{1} R, \ldots, R^{t} X_{k} R\right) .
\end{array}
$$

The ring of invariants $\mathbb{R}\left[x_{i j}^{h}\right]^{O(d)}$ is the ring of polynomial functions $f \in \mathbb{R}\left[x_{i j}^{h}\right]$ which remain invariant under the action (2.3). Its homogeneous component of degree $e$ is denoted by $\mathbb{R}\left[x_{i j}^{h}\right]_{e}^{O(d)}$. Classical invariant theory provides us with a complete description of the multilinear orthogonal invariant of $2 k$ vectors (see [64]). From this it follows that every orthogonal invariant of $\mathbb{R}\left[x_{i j}^{h}\right]^{O(d)}$ is a polynomial in the elements $\operatorname{Tr}\left(U^{i_{1}} U^{i_{2}} \ldots U^{i_{s}}\right)$ where $U^{i}=M^{i}$ or $U^{i}=\left(M^{i}\right)^{t}$ (see [50]). In particular writing $\mathbf{M}=\left(M^{1}, \ldots, M^{k}\right)$, we have

Theorem 2.1. Any invariant 2-form $F$ on $\mathcal{M}^{k}(d, d)$, i.e. any element of $\mathbb{R}\left[x_{i j}^{h}\right]_{2}^{O(d)}$, can be written as follows

$$
F(\boldsymbol{M})=\sum_{i, j=1}^{k} a_{i j} \operatorname{Tr}\left(M^{i}\left(M^{j}\right)^{t}\right)+\sum_{i, j=1}^{k} b_{i j} \operatorname{Tr}\left(M^{i} M^{j}\right)-\sum_{i, j=1}^{k} c_{i j} \operatorname{Tr} M^{i} \operatorname{Tr} M^{j}
$$

We define the symmetric $k \times k$ real matrices $A, B$ and $C$ with entries $(A)_{i j}=a_{i j},(B)_{i j}=b_{i j}$ and $(C)_{i j}=c_{i j}$, respectively and note that any invariant 2 -form $F$ is in a one to one correspondence with the triple of symmetric matrices $(A, B, C)$.

Therefore $F$ depends exactly on three symmetric $k \times k$ matrices $A, B$ and $C$. In particular $\mathbb{R}\left[x_{i j}^{h}\right]_{2}^{O(d)}$ has dimension $\frac{3 k(k+1)}{2}$.

Definition 2.2. We will say that an invariant 2-form $F$ on $\mathcal{M}^{k}(d, d)$ is associated to $(A, B, C)$ when $F$ has the representation given in (2.4).

Remark 2.3. With the language of Definition 2.2, the forms defined in (1.25) are associated with the triples $(A, B, C)=\left(\frac{r}{2} H, \frac{r}{2} H, H\right)$ if $r \geq 2$ and to the triple $(A, B, C)=(0, H, H)$ if $r=1$.

The next remark will be used many times in the paper.

Remark 2.4. The map $F \rightarrow\left(A_{F}, B_{F}, C_{F}\right)$ defined in Definition 2.2 is linear:

$$
\left(A_{F+F^{\prime}}, B_{F+F^{\prime}}, C_{F+F^{\prime}}\right)=\left(A_{F}+A_{F^{\prime}}, B_{F}+B_{F^{\prime}}, C_{F}+C_{F^{\prime}}\right) .
$$

For our computations the basis $\left\{\operatorname{Tr}\left(M^{i}\left(M^{j}\right)^{t}\right), \operatorname{Tr}\left(M^{i} M^{j}\right), \operatorname{Tr} M^{i} \operatorname{Tr} M^{j}\right\}$ of $\mathbb{R}\left[x_{i j}^{h}\right]_{2}^{O(d)}$ provided by Theorem 2.4 can be conveniently replaced by a different bases which takes into account the decomposition of $\mathcal{M}(d, d)$ under the action of $O(d)$.

Let $\mathbb{P}_{i}: \mathcal{M}(d, d) \rightarrow \mathcal{M}(d, d)$ be defined by

$$
\mathbb{P}_{1} M:=\frac{\operatorname{Tr}(M)}{d} \cdot I d(d), \mathbb{P}_{2} M:=\frac{1}{2}\left(M+M^{t}\right)-\mathbb{P}_{1} M, \mathbb{P}_{3} M:=\frac{1}{2}\left(M-M^{t}\right)
$$

and let $\mathbb{P}_{i} \mathcal{M}$ be the image of $\mathbb{P}_{i}$. The $\mathbb{P}_{i}$ are mutually orthogonal projectors and give rise to the orthogonal splitting

$$
\mathcal{M}(d, d)=\mathbb{P}_{1} \mathcal{M} \oplus \mathbb{P}_{2} \mathcal{M} \oplus \mathbb{P}_{3} \mathcal{M}
$$


which is a splitting into irreducible subspaces under the action (2.1) of the orthogonal group. We note, for future reference, that

$$
\operatorname{dim}\left(\mathbb{P}_{1}\right)=1, \quad \operatorname{dim}\left(\mathbb{P}_{2}\right)=\frac{d(d+1)}{2}, \quad \operatorname{dim}\left(\mathbb{P}_{3}\right)=\frac{d(d-1)}{2}
$$

Remark 2.5. The vector spaces of this splitting are irreducible $O(d)$ modules. They are also irreducible $S O(d)$ modules, except for $d=2$ and $d=4$. For $d=2$ the traceless symmetric matrices are reducible over $\mathbb{C}$ and irreducible over $\mathbb{R}$. For $d=4$ the antisymmetric matrices are reducible over $\mathbb{C}$ and $\mathbb{R}$ (see [64], Th. $5.9 \mathrm{~A}$ ).

We will not pursue here the goal of studying 2 -forms which are invariant with respect to the action of $S O(d)$ rather than $O(d)$. This topic is discussed in [51].

Elements of $\mathbb{P}_{1} \mathcal{M}$ are matrices which are multiple of the identity and therefore sometimes called scalar matrices. Similarly $\mathbb{P}_{2} \mathcal{M}$ is called the space of traceless symmetric matrices and $\mathbb{P}_{3} \mathcal{M}$ the space of antisymmetric matrices, and we have

$$
\mathbb{P}_{1} M^{t}=\mathbb{P}_{1} M, \quad \mathbb{P}_{2} M^{t}=\mathbb{P}_{2} M, \quad \mathbb{P}_{3} M^{t}=-\mathbb{P}_{3} M .
$$

We have the following obvious but extremely useful identities:

$$
\begin{gathered}
\langle M, N\rangle:=\operatorname{Tr}\left(M N^{t}\right)=\left\langle\mathbb{P}_{1} M, \mathbb{P}_{1} N\right\rangle+\left\langle\mathbb{P}_{2} M, \mathbb{P}_{2} N\right\rangle+\left\langle\mathbb{P}_{3} M, \mathbb{P}_{3} N\right\rangle, \\
\operatorname{Tr}(M N)=\left\langle\mathbb{P}_{1} M, \mathbb{P}_{1} N\right\rangle+\left\langle\mathbb{P}_{2} M, \mathbb{P}_{2} N\right\rangle-\left\langle\mathbb{P}_{3} M, \mathbb{P}_{3} N\right\rangle, \\
\operatorname{Tr}(M) \operatorname{Tr}(N)=d\left\langle\mathbb{P}_{1} M, \mathbb{P}_{1} N\right\rangle .
\end{gathered}
$$

The next corollary is now immediate and gives another basis of $\mathbb{R}\left[x_{i j}^{h}\right]_{2}^{O(d)}$.

Corollary 2.6. Any invariant 2-form $F$, can be written as follows

$$
F(\boldsymbol{M})=\sum_{i, j=1}^{k}\left\{\left[a_{i j}+b_{i j}-d c_{i j}\right]\left\langle\mathbb{P}_{1} M^{i}, \mathbb{P}_{1} M^{j}\right\rangle+\left[a_{i j}+b_{i j}\right]\left\langle\mathbb{P}_{2} M^{i}, \mathbb{P}_{2} M^{j}\right\rangle+\left[a_{i j}-b_{i j}\right]\left\langle\mathbb{P}_{3} M^{i}, \mathbb{P}_{3} M^{j}\right\rangle\right\}
$$

Proof. Use Theorem 2.1 and the identities (2.6), (2.10) and (2.11).

It is useful and interesting to point out that the forms

$$
\mathcal{F}_{H}^{(r)}(\mathbf{M})=\sum_{i, j=1}^{k} h_{i j} \mathcal{G}_{r}\left(M^{i}, M^{j}\right), \quad r \in\{1, \ldots, d\}
$$

already defined in (1.25), take an especially simple form if described in the language of the projectors $\mathbb{P}_{i}$.

Indeed one has

$$
\begin{gathered}
\mathcal{G}_{r}(M, N)=-(d-r)\left\langle\mathbb{P}_{1} M, \mathbb{P}_{1} N\right\rangle+r\left\langle\mathbb{P}_{2} M, \mathbb{P}_{2} N\right\rangle \quad \text { if } \quad 2 \leq r, \\
\mathcal{G}_{1}(M, N)=-(d-1)\left\langle\mathbb{P}_{1} M, \mathbb{P}_{1} N\right\rangle+\left\langle\mathbb{P}_{2} M, \mathbb{P}_{2} N\right\rangle-\left\langle\mathbb{P}_{3} M, \mathbb{P}_{3} N\right\rangle .
\end{gathered}
$$

Remark 2.7. The expression (2.15) shows that if $r \geq 2$ the extremal form does not depend on the antisymmetric part of the matrices. Using Theorem 1.15, it is easy to conclude that any invariant rank- $r$ convex 2 form is actually convex in the subspace

$$
\overbrace{\mathbb{P}_{3} \mathcal{M} \times \cdots \times \mathbb{P}_{3} \mathcal{M}}^{k-\text { times }}
$$

We will return on this issue in Section 4.

We will use the invariance of the 2 -forms to give rank- $r$ convexity a particularly simple form. To this end the following definitions are crucial. 
Definition 2.8. We define $\mathcal{M}_{d, r}^{k}$ to be the set of elements $\mathbf{M}=\left(M_{1}, \ldots, M_{k}\right)$ of $\mathcal{M}^{k}(d, d)$ such that each $M_{i}$ has the last $d-r$ rows equal to zero. The space $\mathcal{M}_{d, r}^{k}$ is obviously isomorphic to $\mathcal{M}^{k}(d, r)$. When $k=1$, we write $\mathcal{M}(d, r)$ for $\mathcal{M}^{1}(d, r)$ and $\mathcal{M}_{d, r}$ for $\mathcal{M}_{d, r}^{1}$.

Definition 2.9. For $\mathbf{M}=\left(M^{1}, M^{2}, \ldots, M^{k}\right) \in \mathcal{M}^{k}(d, d)$, we define $\mathcal{S}^{k}(d, r)$ by

$$
\mathbf{M} \in \mathcal{S}^{k}(d, r) \Longleftrightarrow \operatorname{dim}\left(\bigcap_{i=1}^{k} \operatorname{Ker}\left(M^{i}\right)\right) \geq d-r .
$$

Remark 2.10. The crucial fact is that

$$
\mathbf{M} \in \mathcal{S}^{k}(d, r) \Longleftrightarrow \exists R \in O(d):\left(R M^{1} R^{t}, \ldots, R M^{k} R^{t}\right) \in \mathcal{M}_{d, r}^{k}
$$

i.e. the map

$$
O(d) \times \mathcal{M}_{d, r}^{k} \rightarrow \mathcal{S}^{k}(d, r)
$$

which sends $(R, \mathbf{M})$ to $R \mathbf{M} R^{t}$ is onto. In short: the orbit of $\mathcal{M}_{d, r}^{k}$ under the action of $O(d)$ is $\mathcal{S}^{k}(d, r)$.

Definition 2.11. Given an invariant 2-form $F$ over $\mathcal{M}^{k}(d, d)$ we denote by $F \downarrow$ its restriction to $\mathcal{S}^{k}(d, r)$ and by $F \Downarrow$ its restriction to $\mathcal{M}_{d, r}^{k}$. We call $F \Downarrow$ the restricted form associated to $F$.

Since $F$ is $O(d)$-invariant, $F\left(R^{t} \mathbf{M} R\right)=F(\mathbf{M})$ for any $R \in O(d)$ hence, by (2.19), the values of $F \downarrow$ are completely determined by the values of $F \Downarrow$.

We introduce the following notations:

Definition 2.12. Given a jointly rank- $r$ convex, invariant 2 -form $F$ we set

$$
\left\{\begin{array}{l}
N_{F} \equiv \text { Eigenspace relative to negative eigenvalues of } F \\
K_{F} \equiv \text { Eigenspace relative to null eigenvalues of } F \\
P_{F} \equiv \text { Eigenspace relative to positive eigenvalues of } F \\
K_{\models} \equiv\left\{\mathbf{M} \in \mathcal{S}^{k}(d, r): F(\mathbf{M})=0\right\} \\
P_{F} \equiv\left\{\mathbf{M} \in \mathcal{S}^{k}(d, r): F(\mathbf{M})>0\right\} \\
K_{F \downarrow} \equiv\left\{\mathbf{M} \in \mathcal{M}_{d, r}^{k}: F(\mathbf{M})=0\right\} \\
P_{F \downarrow} \equiv\left\{\mathbf{M} \in \mathcal{M}_{d, r}^{k}: F(\mathbf{M})>0\right\} .
\end{array}\right.
$$

Remark 2.13. Note that

$$
\left\{\begin{array}{l}
P_{F}=\left(N_{F} \oplus K_{F}\right)^{\perp} \\
P_{F}=\mathcal{S}^{k}(d, r) \backslash K_{F} \\
P_{F \downarrow}=\mathcal{M}_{d, r}^{k} \backslash K_{F \downarrow} .
\end{array}\right.
$$

Remark 2.14. The sets $K_{F}$ and $P_{F}$ are completely determined by $K_{F \downarrow}$ and $P_{F \downarrow}$ respectively. In fact the sets $K_{\mp \downarrow}$ and $P_{F}$ are the orbits of the sets $K_{F \downarrow}$ and $P_{F \downarrow}$ under the action of $O(d)$, i.e. the map (2.19) restricted to $O(d) \times K_{F \downarrow}\left(\right.$ resp. $\left.O(d) \times P_{F \downarrow}\right)$ surjects onto $O(d) \times K_{\mp \downarrow}\left(\right.$ resp. $\left.O(d) \times P_{F}\right)$.

Finally we introduce some notations to be used in the next section. For any matrix $M \in \mathcal{M}_{d, r}$, we define (see (2.22)) $N \in \mathcal{M}(r, r)$ to be its principal minor, obtained by taking only the first $r$ rows and columns of $M$, and $E \in \mathcal{M}(r, d-r)$ the matrix formed with the first $r$ rows and the last $(d-r)$ columns of $M$.

$$
M=\left(\begin{array}{c|c}
N & E \\
\hline 0 & 0
\end{array}\right)
$$

with $N \in \mathcal{M}(r, r)$ and $E \in \mathcal{M}(r, d-r)$. 
The following relations link the invariants of $M$ to those of $N$ and $E$ :

$$
\begin{aligned}
\operatorname{Tr}\left(M M^{t}\right) & =\operatorname{Tr}\left(N N^{t}\right)+\operatorname{Tr}\left(E E^{t}\right) \\
\operatorname{Tr}(M M) & =\operatorname{Tr}(N N) \\
(\operatorname{Tr} M)^{2} & =(\operatorname{Tr} N)^{2} \\
(\operatorname{Tr} N)^{2} & =r\left|\mathbb{P}_{1} N\right|^{2} .
\end{aligned}
$$

With a slight abuse of notation, we have denoted by $\mathbb{P}_{1} N$ the first canonical projection acting on the space $\mathcal{M}(r, r)$ (rather than on the space $\mathcal{M}(d, d)$ ).

\section{An ALGEBRAIC CHARACTERIZATION OF RANK-r CONVEXITY}

The present section is devoted to prove Theorems 1.15 and 1.20. The most important ingredients are Lemma 3.1, Corollary 3.2 and Lemma 3.3. We use the notation of Section 2.

Lemma 3.1. Let $d \geq 2$. Let $F$ be an invariant 2-form associated to the triple $(A, B, C)$ in the sense of Definition 2.2. For any integer $r \in[2, d]$, the following characterization holds:

$$
\text { If } r \geq 2, F \text { is jointly rank-r convex } \Longleftrightarrow\left\{\begin{array}{l}
A+B-r C \geq 0 \\
A+B \geq 0 \\
A-B \geq 0
\end{array}\right.
$$

and

$$
F \text { is jointly rank-1 convex } \Longleftrightarrow\left\{\begin{array}{l}
A \geq 0 \\
A+B-C \geq 0 .
\end{array}\right.
$$

Proof of (3.1).

Part 1: $r=d$. We want to check the joint convexity of $F$. This is an immediate consequence of Corollary 2.6. Indeed, in the new coordinates inherited by the irreducible decomposition of Section 2, (see 2.6), the Hessian of $F$ is the direct sum of the matrices $A+B-d C, A+B$ and $A-B$ each one with its multiplicity. They are the dimensions of $\mathbb{P}_{1} \mathcal{M}, \mathbb{P}_{2} \mathcal{M}$ and $\mathbb{P}_{3} \mathcal{M}$ as given by (2.8).

Part 2: $2 \leq r<d$. We may assume $d \geq 3$, otherwise there is nothing to prove. We need to check the joint rank- $r$ convexity of $F$, with $r \in[2, d-1]$. We divide the proof in several steps.

Step 1. We use (2.7), (2.9) and (2.11). By Remark 2.10, $F$ is jointly rank-r convex on $\mathcal{M}^{k}(d, d)$ if and only if $F \Downarrow$ is jointly convex on $\mathcal{M}_{d, r}^{k}$.

Step 2. Using (2.23), we compute the restriction $F \Downarrow$ of $F$ to $\mathcal{M}_{d, r}^{k}$. One has

$$
\begin{aligned}
F \Downarrow\left(N^{1}, N^{2}, \ldots, N^{k}, E^{1}, E^{2}, \ldots, E^{k}\right)=\sum_{i, j=1}^{k} a_{i j}\left\{\operatorname{Tr}\left(N^{i}\left(N^{j}\right)^{t}\right)\right. & \left.+\operatorname{Tr}\left(E^{i}\left(E^{j}\right)^{t}\right)\right\} \\
& +\sum_{i, j=1}^{k} b_{i j} \operatorname{Tr}\left(N^{i} N^{j}\right)-\sum_{i, j=1}^{k} c_{i j} \operatorname{Tr} N^{i} \operatorname{Tr} N^{j} .
\end{aligned}
$$

Step 3. Rewrite the above expression using the projectors $\mathbb{P}_{i}$ defined in (2.6). Recall that they now act on $r \times r$ matrices. In particular, one has

$$
(\operatorname{Tr} N)^{2}=r\left|\mathbb{P}_{1}\right|^{2}
$$


We get

$$
\begin{gathered}
F \Downarrow\left(N^{1}, N^{2}, \ldots, N^{k}, E^{1}, E^{2}, \ldots, E^{k}\right)=\sum_{i, j=1}^{k}\left[a_{i j}+b_{i j}-r c_{i j}\right]\left\langle\mathbb{P}_{1} N^{i}, \mathbb{P}_{1} N^{j}\right\rangle \\
+\sum_{i, j=1}^{k} a_{i j} \operatorname{Tr}\left(E^{i}\left(E^{j}\right)^{t}\right)+\sum_{i, j=1}^{k}\left[a_{i j}+b_{i j}\right]\left\langle\mathbb{P}_{2} N^{i}, \mathbb{P}_{2} N^{j}\right\rangle \\
+\sum_{i, j=1}^{k}\left[a_{i j}-b_{i j}\right]\left\langle\mathbb{P}_{3} N^{i}, \mathbb{P}_{3} N^{j}\right\rangle .
\end{gathered}
$$

Using Part 1 of the present Theorem applied for $d=r$ and the independence of the $E^{i}$ s from the $N^{i}$ s, we get that (when $r \geq 2$ )

$$
F \Downarrow \text { is jointly convex on } \mathcal{M}_{d, r}^{k} \Longleftrightarrow\left\{\begin{array}{l}
A \geq 0 \\
A+B-r C \geq 0 \\
A+B \geq 0 \\
A-B \geq 0 .
\end{array}\right.
$$

Step 4. Finally we remark that the third and the fourth inequalities in (3.6), imply the first one. On use of Step 1, this concludes Part 2 for the case $d \geq 3$.

Proof of (3.2). The proof is very similar to that of (3.1) except for the fact that when $r=1$ the orthogonal splitting (2.7) trivializes. Only $\mathbb{P}_{1}$ survives. One is then led to the system of inequalities in (3.6) but without the third and fourth inequalities (which were originated by the projections $\mathbb{P}_{2}$ and $\mathbb{P}_{3}$ ). We get that

$$
F \Downarrow \text { is jointly convex on } \mathcal{M}_{d, 1}^{k} \Longleftrightarrow\left\{\begin{array}{l}
A \geq 0 \\
A+B-C \geq 0 .
\end{array}\right.
$$

This time, however, the first inequality is not automatically satisfied and it must be retained. The proof is now complete.

The following corollary is particularly useful. It performs the first step of our program. It shows that the forms defined in (1.25), are indeed rank- $r$ convex.

Corollary 3.2. For each $r=1,2, \ldots(d-1)$, the form $\mathcal{F}_{H}^{(r)}$ defined in $(1.25)$ is rank-r convex.

Proof. Let us begin with the case $r \geq 2$. We need to verify (3.1). Recalling Definition 2.2 and Remark 2.3 , the forms $\mathcal{F}_{H}^{(r)}$ are associated to the triple $(A, B, C)=\left(\frac{r}{2} H, \frac{r}{2} H, H\right)$. Hence, by Lemma $3.1, \mathcal{F}_{H}^{(r)}$ is rank $r$-convex if and only if

$$
r H \geq 0 .
$$

By assumption $r \geq 2$, and $H \geq 0$. Hence (3.8) is satisfied. The case $r=1$ is similar. This time $\mathcal{F}_{H}^{(1)}$ is associated to the triple $(A, B, C)=(0, H, H)$. Hence, by Lemma $3.1, \mathcal{F}_{H}^{(1)}$ is rank 1-convex.

We now perform the second part of our program addressing the extremality issue.

Lemma 3.3. The forms $(1.25)$ are $(r, s)$ extremal for $r=1,2, \ldots,(d-1), s=r+1, \ldots, d$.

Proof. We start by considering the case $r \geq 2$. For fixed $r$, we need to show that, if $\mathcal{F}_{H}^{(r)}$ is the sum of a jointly rank- $r$ convex form $\mathcal{Q}$ and a jointly rank- $s$ convex form $\mathcal{S}$, then $\mathcal{S}$ is zero. Recall that, by assumption, $\mathcal{S}$ is 
rank- $s$ convex and $s>r>1$. Hence, according to Definition 2.2, it is associated to a triple $\left(A_{S}, B_{S}, C_{S}\right)$ which, by Lemma 3.1, satisfies the following system of inequalities:

$$
\left\{\begin{array}{l}
A_{S}+B_{S}-s C_{S} \geq 0 \\
A_{S}+B_{S} \geq 0 \\
A_{S}-B_{S} \geq 0
\end{array}\right.
$$

The form $\mathcal{F}_{H}^{(r)}$ is associated, by construction, to the triple $(A, B, C)=\left(\frac{r}{2} H, \frac{r}{2} H, H\right)$ (see Rem. 2.3). Since $\mathcal{F}_{H}^{(r)}=\mathcal{Q}+\mathcal{S}$, the form $\mathcal{Q}$ is associated with the triple $\left(A_{Q}, B_{Q}, C_{Q}\right)$ given by

$$
\left\{\begin{array}{l}
A_{Q}=\frac{r}{2} H-A_{S} \\
B_{Q}=\frac{r}{2} H-B_{S} \\
C_{Q}=H-C_{S}
\end{array}\right.
$$

By Lemma 3.1, the form $\mathcal{Q}$ is rank- $r$ convex if and only if

$$
\left\{\begin{array}{l}
A_{Q}+B_{Q}-r C_{Q}:=r H-r H-A_{S}-B_{S}+r C_{S} \geq 0 \Leftrightarrow A_{S}+B_{S} \leq r C_{S} \\
A_{Q}+B_{Q}:=r H-\left(A_{S}+B_{S}\right) \geq 0 \\
A_{Q}-B_{Q}:=B_{S}-A_{S} \geq 0
\end{array}\right.
$$

The third inequality of (3.9), together with the third inequality of (3.11), implies $B_{S}=A_{S}$. The remaining inequalities read as follows:

$$
\left\{\begin{array}{l}
2 A_{S}-s C_{S} \geq 0 \\
2 A_{S} \geq 0 \\
2 A_{S} \leq r C_{S} \\
r H-2 A_{S} \geq 0
\end{array}\right.
$$

which imply

$$
\left\{\begin{array}{l}
r C_{S} \geq 2 A_{S} \\
A_{S} \geq 0 \\
(r-s) C_{S} \geq 0
\end{array}\right.
$$

Since $r<s$, the third inequality implies $C_{S} \leq 0$. The first and the second imply $C_{S} \geq 0$, and therefore $C_{S}=0$. Then the first and second inequality in (3.13) implies $A_{S}=0$. Therefore the rank- $s$ convex form $\mathcal{S}$ is associated to the triple $(0,0,0)$, and therefore it is identically zero as asserted. This completes the proof for the case $r \geq 2$.

The case $r=1$ is similar. Suppose $\mathcal{F}_{H}^{(1)}=\mathcal{Q}+\mathcal{S}$ where $\mathcal{Q}$ is jointly rank-1 convex and $\mathcal{S}$ is jointly rank- $s$ convex. Let $\left(A_{Q}, B_{Q}, C_{Q}\right)$ and $\left(A_{S}, B_{S}, C_{S}\right)$ be associated to $\mathcal{Q}$ and $\mathcal{S}$ respectively. Recall that by $(2.3), \mathcal{F}_{H}^{(1)}$ is associated to $(0, H, H)$. We have

$$
\begin{array}{r}
A_{S}-B_{S} \geq 0, \\
A_{S}+B_{S} \geq 0, \\
A_{S}+B_{S}-s C_{S} \geq 0,
\end{array}
$$

and, by construction,

$$
\begin{gathered}
A_{Q}=-A_{S}, \\
B_{Q}=H-B_{S}, \\
C_{Q}=H-C_{S} .
\end{gathered}
$$


By Lemma 3.1

$$
\begin{aligned}
A_{Q} & \geq 0, \\
A_{Q}+B_{Q}-C_{Q} & \geq 0 .
\end{aligned}
$$

Note that (3.14) and (3.15) imply $A_{S} \geq 0$. Using the latter and (3.17) we get $A_{Q} \leq 0$. The latter and (3.20) imply $A_{Q}=0$. Then by (3.17) we get $A_{S}=0$. Now use (3.14) and (3.15) to obtain $B_{S}=0$. Since $A_{S}=B_{S}=0$ and $s \geq 0,(3.16)$ implies $C_{S} \leq 0$; since $B_{S}=0$, by (3.18) we get $B_{Q}=H$. We are left with the inequalities

$$
\left\{\begin{array}{cl}
C_{S} & \leq 0 \\
H-C_{Q}=C_{S} & \geq 0
\end{array}\right.
$$

from which we conclude $C_{S}=0$. Thus $A_{S}=B_{S}=C_{S}=0$, hence $\mathcal{S}=0$.

We are now ready to prove Theorem 1.15.

Proof of Theorem 1.15.

Part I. Fix $r \geq 2$. By Corollary 3.2 we know that $F=\mathcal{F}_{H}^{(r)}$ is rank- $r$ convex. By Lemma 3.3, we also know that the form $\mathcal{F}_{H}^{(r)}$ is $(r, s)$ extremal for $s>r$. Therefore to prove Part I, it is enough to show that for any jointly invariant rank- $r$ convex 2 -form $F$, there exists a symmetric matrix $H$ such that, $F$ can be written as the sum of $\mathcal{F}_{H}^{(r)}$ and a jointly convex invariant 2 -form $\mathcal{C}_{H}$.

Let $F$ be associated to the triple $\left(A_{F}, B_{F}, C_{F}\right)$. Set

$$
H:=\frac{A_{F}+B_{F}}{r}
$$

and define $\mathcal{F}_{H}^{(r)}$ accordingly. Thus $\mathcal{F}_{H}^{(r)}$ is associated to

$$
\left(\frac{A_{F}+B_{F}}{2}, \frac{A_{F}+B_{F}}{2}, \frac{A_{F}+B_{F}}{r}\right) .
$$

Since $F$ is rank- $r$ convex, the second inequality in (3.1) shows that $H$, as defined in (3.23) is non negative and therefore $\mathcal{F}_{H}^{(r)}$ is $(r, s)$ extremal by Lemma 3.3. It remains to show that $\mathcal{C}:=F-\mathcal{F}_{\left(A_{F}+B_{F}\right) / r}^{(r)}$ is convex. By construction, $\mathcal{C}$ is associated to the triple

$$
\left(A_{F}, B_{F}, C_{F}\right)-\left(\frac{A_{F}+B_{F}}{2}, \frac{A_{F}+B_{F}}{2}, \frac{A_{F}+B_{F}}{r}\right)=\left(\frac{A_{F}-B_{F}}{2}, \frac{B_{F}-A_{F}}{2}, C_{F}-\frac{A_{F}+B_{F}}{r}\right) .
$$

By Lemma 3.1, $\mathcal{C}$ is convex if and only if the following inequalities hold

$$
\begin{aligned}
\frac{A_{F}-B_{F}}{2}+\frac{B_{F}-A_{F}}{2}-d\left(C_{F}-\frac{A_{F}+B_{F}}{r}\right) & \geq 0, \\
& \frac{A_{F}-B_{F}}{2}+\frac{B_{F}-A_{F}}{2} \geq 0, \\
& \frac{A_{F}-B_{F}}{2}-\frac{B_{F}-A_{F}}{2} \geq 0 .
\end{aligned}
$$

The latter holds if and only if

$$
\begin{array}{r}
A_{F}+B_{F}-r C_{F} \geq 0, \\
A_{F}-B_{F} \geq 0,
\end{array}
$$


which are verified because of the first and third inequalities in (3.1).

Part II. We assume $k \geq 2$ since for $k=1$ there is nothing to prove. Given $r \geq 2$, let $F=\mathcal{F}_{H}^{(r)}$ for some symmetric $H$ such that $H \geq 0$. We first prove that if $H$ has rank one, then $F$ is $(r, s)$ extremal for $s=r, \ldots, d$. Assume by contradiction that $F=\mathcal{Q}+\mathcal{P}$ with $\mathcal{Q}$ and $\mathcal{P}$ non zero, linearly independent and $(r, s)$ extremal for $s=r, \ldots, d$. Here in particular $\mathcal{Q}$ and $\mathcal{P}$ must be $(r, s)$ extremal for $s>r$. Hence there exist $H_{Q}$ and $H_{P}$ symmetric and non negative definite such that $\mathcal{Q}$ and $\mathcal{P}$ are associated to $\left(\frac{r}{2} H_{Q}, \frac{r}{2} H_{Q}, H_{Q}\right),\left(\frac{r}{2} H_{P}, \frac{r}{2} H_{P}, H_{P}\right)$, respectively. Since $\mathcal{Q} \neq 0, \mathcal{P} \neq 0, \operatorname{rank}\left(H_{Q}\right) \geq 1$ and $\operatorname{rank}\left(H_{P}\right) \geq 1$. Since $\mathcal{Q}$ and $\mathcal{P}$ are linearly independent, $\operatorname{rank}\left(H_{P}+H_{Q}\right) \geq 2$. By construction, $H=H_{P}+H_{Q}$ and we obtain a contradiction because $\operatorname{rank}(H)=1$ by assumption.

Now we prove that if $r_{H}=\operatorname{rank}(H) \geq 2, \mathcal{F}_{H}^{(r)}$ is not $(r, r)$ extremal. Indeed, if $\mathcal{F}_{H}^{(r)}$ is associated to $\left(\frac{r}{2} H, \frac{r}{2} H, H\right)$ we write $H=\sum_{i=1}^{r_{H}} H_{i}$ with $H_{i}=h_{i} \otimes h_{i}, h_{i} \neq 0$. Then $\mathcal{F}^{(r)}=\sum_{i=1}^{r_{H}} \mathcal{F}_{H_{i}}^{(r)}, \mathcal{F}_{H_{i}}^{(r)}$ is $(r, r)$ extremal by the first part and, if $i \neq j$, they are linearly independent by construction. Hence $\mathcal{F}_{H}^{(r)}$ is not $(r, r)$ extremal if $r \geq 2$.

Remark 3.4. Inspection of the proof of Theorem 1.15 shows an algorithm which, for any invariant rank- $r$ convex 2-form $F$ associated to $(A, B, C)$, delivers a specific extremal invariant rank- $r$ convex 2 -form namely $\mathcal{F}_{\underline{\underline{A+B}}}^{(r)}$ and, by setting

$$
\mathcal{C}:=F-\mathcal{F}_{\frac{A+B}{r}}^{(r)}
$$

it also delivers an explicit convex part in the decomposition (1.26).

The latter decomposition, as already remarked in Remark 1.22, is not unique but it has the following characteristic property. For all $\epsilon>0$ and for all $G=G^{t} \geq 0$, the 2 -form $F-\mathcal{F}_{\frac{A+B}{r}+\epsilon G}^{(r)}$ is not convex!

Exactly the same observation and the same formula applies to the case $r=1$.

Proof of Theorem 1.16.

Part I. By Corollary 3.2 and Lemma 3.3, the form (1.23) is jointly rank-1 and quadratically $(1, s)$ extremal for $s>1$. Therefore, in order to prove Part I, it is enough to show that for any jointly invariant rank-1 convex 2-form $F$, there exists a symmetric matrix $H$ such that $F$ can be written as the sum of $\mathcal{F}_{H}^{(1)}$ and a jointly convex invariant 2-form $\mathcal{C}$. Let $F$ be associated to the triple $\left(A_{F}, B_{F}, C_{F}\right)$ and set $H=A_{F}+B_{F}$. By Remark 2.3, $\mathcal{F}_{H}^{(1)}$ is associated to $(0, H, H)$, hence $\mathcal{C}$ is associated to the triple

$$
\left(A_{S}, B_{S}, C_{S}\right)=\left(A_{F},-A_{F}, C_{F}-A_{F}-B_{F}\right) .
$$

By assumption, $F$ is jointly rank 1-convex, hence, by Lemma 3.1

$$
\left\{\begin{array}{l}
A_{F} \geq 0 \\
A_{F}+B_{F}-C_{F} \geq 0 .
\end{array}\right.
$$

We need to prove that for all integers $s \in\{2, d\}$ one has

$$
\left\{\begin{array}{l}
A_{S}+B_{S}-s C_{S} \geq 0 \\
A_{S}+B_{S} \geq 0 \\
A_{S}-B_{S} \geq 0 .
\end{array}\right.
$$

By (3.30), we have that (3.32) is equivalent to

$$
\left\{\begin{array}{l}
-s\left(C_{F}-A_{F}-B_{F}\right) \geq 0 \\
0 \geq 0 \\
2 A_{F} \geq 0
\end{array}\right.
$$


which is verified by (3.31), for any integer $s \in\{2, d\}$.

Part II. Let $H=\sum_{i=1}^{k} H_{i}, \operatorname{rank}(H)=k, \operatorname{rank}\left(H_{i}\right)=1$. Since $(0, H, H)=\sum_{i=1}^{k}\left(0, H_{i}, H_{i}\right)$ then, by Remark 2.4, $\mathcal{F}_{H}^{(1)}=\sum_{i=1}^{k} \mathcal{F}_{H_{i}}^{(1)}$. By Part I, each $\mathcal{F}_{H_{i}}^{(1)}$ is rank-1 convex and extremal. Hence $F$ is extremal if and only if $F=\mathcal{F}_{H}^{(1)}$ with $\operatorname{rank}(H)=1$.

Proof of Proposition 1.19. We treat the case $r \geq 2$ first. Let $F$ be associated to $\left(A_{F}, B_{F}, C_{F}\right)$. In the proof of Theorem 1.15, Part I, we already proved that $F=\mathcal{F}_{H_{F}}^{(r)}+\mathcal{C}$ where $H_{F}=\left(A_{F}+B_{F}\right) / r$ and $\mathcal{C}$ is associated to

$$
\left(\frac{A_{F}-B_{F}}{2}, \frac{B_{F}-A_{F}}{2}, C_{F}-\frac{A_{F}+B_{F}}{r}\right)
$$

and it is convex. Now write $H_{F}=\sum_{j=1}^{k} u_{j}^{F} \otimes u_{j}^{F}$ using the spectral theorem and note that, by Remark 2.4, $\mathcal{F}_{H_{F}}^{(r)}=\sum_{j=1}^{k} \mathcal{F}_{u_{j}^{F} \otimes u_{j}^{F}}^{(r)}$.

The case $r=1$ is similar. In the proof of Theorem 1.16 we have proved that $F=\mathcal{F}_{H_{F}}^{(1)}+\mathcal{C}$ where $\mathcal{C}$ is convex and $H_{F}=A_{F}+B_{F}$. The difference now is that $H_{F}$ is only symmetric but not necessarily non negative. Therefore

$$
H_{F}=\sum_{j=1}^{\operatorname{rank}\left(H_{F}\right)} H_{j}
$$

with $H_{j}$ symmetric and rank one. Hence $H_{j}=\sigma_{j} u^{j} \otimes u^{j}$ where $\sigma^{j}$ is either 1 or -1 . The rest of the proof is identical to the previous case.

Proof of Proposition 1.20. $(\Rightarrow)$ We use the canonical form given by Proposition 1.19. It suffices to prove that $\mathcal{F}_{H}^{(r)}$ is $(r, s)$ extremal for every $s>r$. This was already proved in Lemma 3.3 .

$(\Leftarrow)$ An immediate consequence of the definitions.

\section{Some Characterizations of EXTREMAL FORMS}

In this section we present some characterizations of the extremal forms $\mathcal{F}_{H}^{(r)}$ as defined in (1.25). We treat in detail the case $k=1$ and the cases $k \geq 1$ when $\operatorname{rank} H=1$ and when $\operatorname{rank} H=k$.

We are interested in describing explicitly the spaces in (2.12) only for functions which are $(r, d)$ extremal. As usual the case $r=1$ is special and will be treated separately. We treat the case $k=1$ first since, as we shall see, the case when $F$ is $(r, r)$ extremal is essentially reduced to the case $k=1$.

We shall say that the $(r, d-r)$ block form of a matrix $M$ is a block decomposition of the form

$$
\left(\begin{array}{l|l}
M_{11} & M_{12} \\
\hline M_{21} & M_{22}
\end{array}\right)
$$

where $M_{11} \in \mathcal{M}(r, r), M_{12} \in \mathcal{M}(r, d-r), M_{21} \in \mathcal{M}(d-r, r), M_{22} \in \mathcal{M}(d-r, d-r)$. When there is no risk of ambiguity, we omit the horizontal and vertical segments in (4.1).

Lemma 4.1. Let $k=1, d \geq 3, r \in\{2, \ldots, d\}$ and recall (1.22) and (2.7). Let $F=G^{(r)}$ be the $(r, r)$ extremal rank-r convex form defined by

$$
G^{(r)}(M)=\mathcal{G}_{r}(M, M)=\frac{r}{2}\left(\operatorname{Tr}\left(M M^{t}\right)+\operatorname{Tr}\left(M^{2}\right)\right)-\operatorname{Tr}(M)^{2} .
$$


Then

$$
\begin{aligned}
N_{G^{(r)}} & \equiv \mathbb{P}_{1} \mathcal{M} \\
K_{G^{(r)}} & \equiv \mathbb{P}_{3} \mathcal{M} \\
K_{G^{(r) \Downarrow}} & \equiv \bigcup_{\lambda \in \mathbb{R},}\left(\begin{array}{c|c}
\lambda I d(r)+Q & 0 \\
\hline 0 & 0
\end{array}\right) .
\end{aligned}
$$

For the matrix in (4.5), we have used the $(r, d-r)$ block form defined in (4.1).

Proof. Recall Part 1 of the proof of Lemma 3.1. The Hessian of an invariant rank- $r$ convex two form associated to $(A, B, C)$ in the sense of Definition 2.2, is the direct sum $A+B-d C, A+B$ and $A-B$ with their multiplicities, the matrices above being associated to the spaces $\mathbb{P}_{1} \mathcal{M}, \mathbb{P}_{2} \mathcal{M}$ and $\mathbb{P}_{3} \mathcal{M}$ respectively. In our case $k=1$ and $(A, B, C)$ reduces to the real numbers $\left(\frac{r}{2}, \frac{r}{2}, 1\right)$. Therefore in suitable coordinates the Hessian of $\mathcal{G}_{r}$ is given by

$$
\operatorname{diag}(r-d, \overbrace{r, \ldots, r}^{\frac{d(d+1)}{2} \text { times }}, \overbrace{0, \ldots, 0}^{\frac{d(d-1)}{2} \text { times }})
$$

This proves (4.3) and (4.4). We now prove (4.5). In this case one has $A=a, B=b, C=c$ and

$$
\begin{aligned}
G^{(r)} \Downarrow(N, E)= & (a+b-r c)\left|\mathbb{P}_{1} N\right|^{2}+a|E|^{2}+(a+b)\left|\mathbb{P}_{2} N\right|^{2} \\
& +(a-b)\left|\mathbb{P}_{3} N\right|^{2} \\
= & \frac{r}{2}|E|^{2}+r\left|\mathbb{P}_{2} N\right|^{2}
\end{aligned}
$$

Therefore $G^{(r)} \Downarrow=0$ if and only if $E=0$ and $\mathbb{P}_{2} N=0$, i.e.

$$
M=R^{t}\left(\begin{array}{c|c}
N^{1}+N^{3} & 0 \\
\hline 0 & 0
\end{array}\right) R
$$

for some $N_{1}=\lambda I d(r)$ and $N^{3}=-\left(N^{3}\right)^{t} \in \mathbb{P}_{3} \mathcal{M}$.

Note that, as $R$ ranges over $O(d)$ the set of matrices

$$
R^{t}\left(\begin{array}{c|c}
N^{3} & 0 \\
\hline 0 & 0
\end{array}\right) R
$$

coincides with $\mathbb{P}_{3} \mathcal{M}$ when $d=3, r=2$. In this case our result reduces to that proved in [38], p. 546. However, when $d>3$, this is not generally the case since $3 \times 3$ antisymmetric non zero matrices have rank 2 , while $4 \times 4$ antisymmetric non zero matrices may have rank 2 or 4 .

The next result refers to invariant $(r, r)$ extremal jointly rank- $r$ convex functions, for $r \geq 2$.

Lemma 4.2. Given the integers $k \geq 1, d \geq 3, r \in\{2, \ldots, d\}$ and a symmetric matrix $H \geq 0$, let $F(\mathbf{M})=$ $\mathcal{F}_{H}^{(r)}(\mathbf{M})$ be $(r, r)$ extremal so that there exists a non zero vector $u$ such that $H=u \otimes u$, see (2.14). Use the 
notations of Definition 2.12. One has

$$
\begin{aligned}
M \in N_{F_{H}} & \Longleftrightarrow \sum_{i=1}^{k} u_{i} M^{i} \in \mathbb{P}_{1} \mathcal{M}, \\
M \in K_{F_{H}} & \Longleftrightarrow \sum_{i=1}^{k} u_{i} M^{i} \in \mathbb{P}_{3} \mathcal{M}, \\
M \in K_{F \downarrow} & \Longleftrightarrow \sum_{i=1}^{k} u_{i} M^{i} \in K_{G^{(r)} \Downarrow} .
\end{aligned}
$$

Sketch of the proof. By construction

$$
\mathcal{F}_{H}^{(r)}\left(M^{1}, M^{2}, \ldots, M^{k}\right)=G^{(r)}\left(\sum_{i=1}^{k} u_{i} M^{i}\right)
$$

Then use Lemma 4.1 .

Proposition 4.3. Given three integers $1 \leq k, 3 \leq d$ and $2 \leq r \leq d$, the set of $(r, r)$-extremal, jointly rank- $r$ convex, invariant two forms is characterized by the following property: $F$ is $(r, r)$-extremal if and only if the restriction $F \Downarrow$ of $F$ to $\mathcal{M}_{d, r}^{k}$ maximizes the dimension of its kernel among all possible jointly rank-r convex, invariant two forms which are not convex.

Proof. Let $F$ be a jointly rank- $r$ convex invariant 2 -form associated to $(A, B, C)$. By $(3.5)$, the Hessian of $F \Downarrow$ in suitable coordinates, is given by

$$
(A+B-r C) \oplus\{\underbrace{A \oplus \cdots \oplus A}_{\mathrm{r}(\mathrm{d}-\mathrm{r}) \text { times }}\} \oplus\{\underbrace{(A+B) \oplus \cdots \oplus(A+B)}_{\frac{r^{2}+r}{2}-1 \text { times }}\} \oplus\{\underbrace{(A-B) \oplus \cdots \oplus(A-B)}_{\frac{r^{2}-r}{2} \text { times }}\}
$$

For a $k \times k$ symmetric matrix $M$ we set $z(M)$ to be the number of zero eigenvalues of $M$. With this notation we have

$$
\operatorname{dim}(\operatorname{Ker}(F \Downarrow))=z(A+B-r C)+r(d-r) z(A)+\left(\frac{r^{2}+r}{2}-1\right) z(A+B)+\left(\frac{r^{2}-r}{2}\right) z(A-B) .
$$

Let us compute the dimension of $\operatorname{Ker} F \Downarrow$ when $F$ is $(r, r)$ extremal. In this case $C=H=u \otimes u, A=B=\frac{r}{2} u \otimes u$. Hence

Therefore, when $F=\mathcal{F}_{u \otimes u}$, one has

$$
\left\{\begin{array}{ccccc}
z(A+B-r C) & = & k & = & z(A-B) \\
z(A+B) & = & z(A) & = & k-1
\end{array} .\right.
$$

$$
\begin{aligned}
\Lambda(r, d, k) & :=\operatorname{dim}\left(\operatorname{Ker}\left(\mathcal{F}_{u \otimes u}^{(r)} \Downarrow\right)\right) \\
& =k+r(d-r)(k-1)+\left(\frac{r^{2}+r}{2}-1\right)(k-1)+\left(\frac{r^{2}-r}{2}\right) k .
\end{aligned}
$$

Therefore if $F$ is $(r, r)$ extremal $(r \geq 2)$ then

$$
\operatorname{dim}(\operatorname{Ker}(F \Downarrow))=\Lambda(r, d, k) .
$$

We now show that if $F$ is not convex then

$$
\operatorname{dim}(\operatorname{Ker}(F \Downarrow)) \leq \Lambda(r, d, k) .
$$


Assume, for contradiction, that $F$ is not convex and $\operatorname{dim}(\operatorname{Ker}(F \Downarrow))>\Lambda(r, d, k)$. We claim that necessarily either

$$
z(A)=k \quad \text { or } \quad z(A+B)=k .
$$

Indeed at least one of the addends of the sum in (4.14) must increase; the first and the fourth cannot increase. The second and the third can only increase if $z(A)>k-1$ or $z(A+B)>k-1$ i.e. if and only if (4.15) holds as claimed. We now check that neither case can occur in (4.15).

1. $\mathbf{z}(\mathbf{A})=\mathbf{k}$ cannot occur. Indeed, $z(A)=k$ implies $A=0$, because $A$ is symmetric. Then, since by rank- $r$ convexity $A-B \geq 0$ and $A+B \geq 0$, one has that also $B=0$. Next, since by rank- $r$ convexity $A+B-r C \geq 0$, one obtains $C \leq 0$. In this case, by Lemma 3.1, $F$ is convex, which contradicts the assumption.

2. $\mathbf{z}(\mathbf{A}+\mathbf{B})=\mathbf{d}$ cannot occur. If $z(A+B)=d$, then $A+B=0$. The condition $A+B-r C \geq 0$ implies $C \leq 0$ and, by Lemma $3.1, F$ is convex.

This shows that, if $F$ is not convex, then $\operatorname{dim}(\operatorname{Ker} F \Downarrow) \leq \Lambda(r, d, k)$ and therefore, $\operatorname{dim}(\operatorname{Ker} F \Downarrow)$ is maximal if $F \Downarrow$ is extremal.

Now we want to show that, conversely, if $\operatorname{dim}(\operatorname{Ker} F \Downarrow)=\Lambda(r, d, k)$, then $F$ is $(r, r)$ extremal. Indeed, if $F$ is not convex we have already seen that $\operatorname{dim}(\operatorname{Ker} F \Downarrow) \leq \Lambda(r, d, k), z(A)<k$ and $z(A+B)<k$. Therefore, by (4.14), one necessarily has $z(A-B)=k=z(A+B-r C)$. Since $A, B$ and $C$ are symmetric, this implies $A=B$ and $C=\frac{A+B}{r}$. Setting $H:=C$ one has that $F$ is associated to $\left(\frac{r}{2} H, \frac{r}{2} H, H\right)$ and therefore it is $(r, r+1)$ extremal and is denoted by $\mathcal{F}_{H}^{(r)}$. Recall that $H=0$ implies $\mathcal{F}_{H}^{(r)}=0$ which implies $\mathcal{F}_{H}^{(r)}$ convex, hence $H \neq 0$. Moreover for any non negative $H \neq 0$,

$$
\operatorname{dim} \operatorname{Ker}\left(\mathcal{F}_{H}^{(r)}\right)=k+r(d-r) z(H)+\left(\frac{r^{2}+r}{2}-1\right) z(H)+\frac{r^{2}-r}{2}
$$

The latter is always less than or equal to $\Lambda(r, d, k)$ with equality (for $H \neq 0$ ) if and only if $\operatorname{rank}(H)=1$.

In Lemma 4.1 we have described the spaces $(4.3),(4.4),(4.5)$ for $(r, r)$ extremal invariant 2-forms i.e. forms $\mathcal{F}_{H}^{r}$ with $\operatorname{rank}(H)=1$. Now we want to describe these spaces when $\operatorname{rank}(H)$ is maximal.

We need to recall the following result, due to Von Neumann, see [63].

Theorem 4.4. Let $A, B \in \mathcal{M}(k, k)$ be two symmetric non negative matrices. Let $0 \leq a_{1} \leq a_{2} \leq \cdots \leq a_{k}$ and $0 \leq b_{1} \leq b_{2} \leq \cdots \leq b_{k}$ be the eigenvalues of $A$ and $B$ respectively. Then

$$
a_{1} b_{k}+\cdots+a_{k} b_{1} \leq \operatorname{Tr}(A B) \leq a_{1} b_{1}+\cdots+a_{k} b_{k} .
$$

Since the matrix $R^{t} B R$ has the same eigenvalues as $B$ if $R$ is orthogonal, we have the following corollaries.

Corollary 4.5. Let $A$ and $B$ be as in Theorem 4.4. For each $R \in O(k)$,

$$
a_{1} b_{k}+\cdots+a_{k} b_{1} \leq \operatorname{Tr}\left(A R^{t} B R\right) \leq a_{1} b_{1}+\cdots+a_{k} b_{k}
$$

Corollary 4.6. Let $A, B \in \mathcal{M}(k, k)$ be two symmetric non negative matrices such that $\operatorname{Tr}(A B)=0$. Then $\operatorname{rank}(A)+\operatorname{rank}(B) \leq k$.

Proof. Suppose that $\operatorname{rank}(A)=s$. Let $a_{1}=\cdots=a_{k-s}=0$ and $a_{k+1} \neq 0$. Since

$$
0=\operatorname{Tr}(A B) \geq a_{k-s+1} b_{s}+\cdots+a_{k} b_{1} \geq 0
$$

(the first inequality by Th. 4.4, the second by non negativity of $A$ and $B$ ) we have

$$
a_{k-s+1} b_{s}+\cdots+a_{k} b_{1}=0 .
$$


Hence, since $a_{k-s+1}, \ldots, a_{k}$ are strictly positive, we have $b_{s}=\cdots=b_{1}=0$ and $\operatorname{rank}(B) \leq k-s$, from which the result follows.

Lemma 4.7. Let $F=\mathcal{F}_{H}^{(r)}$ with $\operatorname{rank}(H)=k$. Then

$$
\begin{aligned}
N_{F_{H}} & =\underbrace{\mathbb{P}_{1} \mathcal{M} \oplus \cdots \oplus \mathbb{P}_{1} \mathcal{M}}_{k \text { times }}, \\
K_{F_{H}} & =\underbrace{\mathbb{P}_{3} \mathcal{M} \oplus \cdots \oplus \mathbb{P}_{3} \mathcal{M}}_{k \text { times }}, \\
\operatorname{Ker} F \Downarrow & =K_{G^{(r) \Downarrow} .}
\end{aligned}
$$

Proof. First we prove (4.18) and (4.19). As in the proof of Lemma 4.1 we observe that the Hessian of an invariant 2-form associated to $(A, B, C)$ is the direct sum of $(A+B-d C, A+B, A-B)$ with multiplicities $k$, $k\left(\frac{d(d+1)}{2}-1\right)$ and $k\left(\frac{d(d-1)}{2}\right)$ respectively. For extremal forms with $r \geq 2$, one has that there exists $H$ such that $H=H^{t}, H \geq 0$ and

Hence we get (4.18) and (4.19).

$$
\left\{\begin{array}{ccc}
A+B-d C & = & (r-d) H \\
A+B & = & r H \\
A-B & = & 0
\end{array}\right.
$$

We now prove (4.20). We need to prove that

$$
F \Downarrow\left(N^{1}, \ldots, N^{k}, E^{1}, \ldots, E^{k}\right)=0 \Longleftrightarrow E^{i}=0 \text { and } N^{i} \in \mathbb{P}_{1} \mathcal{M} \oplus \mathbb{P}_{3} \mathcal{M}, i=1, \ldots, k .
$$

By Remark 2.3, the form $F=\mathcal{F}_{H}^{r}$ is associated to $\left(\frac{r}{2} H, \frac{r}{2} H, H\right)$ and by (3.5),

$$
F \Downarrow\left(N^{1}, \ldots, N^{k}, E^{1}, \ldots, E^{k}\right)=\frac{r}{2} \sum_{i, j=1}^{k} h_{i j}\left\langle E^{i}, E^{j}\right\rangle+\sum_{i, j=1}^{k} h_{i j}\left\langle\mathbb{P}_{2} N^{i}, \mathbb{P}_{2} N^{j}\right\rangle .
$$

Therefore (4.22) vanishes if and only if

$$
\left\{\begin{array}{c}
\sum_{i, j=1}^{k} h_{i j}\left\langle E^{i}, E^{j}\right\rangle=0 \\
\sum_{i, j=1}^{k} h_{i j}\left\langle\mathbb{P}_{2} N^{i}, \mathbb{P}_{2} N^{j}\right\rangle=0
\end{array}\right.
$$

If we define the matrices $E$ and $\mathbb{P}_{2} N$ by

$$
E_{i j}=\left\langle E^{i}, E^{j}\right\rangle, \quad\left(\mathbb{P}_{2} N\right)_{i j}=\left\langle\mathbb{P}_{2} N^{i}, \mathbb{P}_{2} N^{j}\right\rangle
$$

then (4.23) can be rewritten as

$$
\left\{\begin{array}{ccc}
\operatorname{Tr}(H E) & = & 0 \\
\operatorname{Tr}\left(H \mathbb{P}_{2} N\right) & = & 0
\end{array}\right.
$$

with $E$ and $\mathbb{P}_{2} N$ symmetric and non negative. Since $\operatorname{rank}(H)=k$, by Corollary 4.6, (4.25) implies $E=0$ and $\mathbb{P}_{2} N=0$. This implies $E^{i}=0$ and $\mathbb{P}_{2} N_{i}=0$ for all $i=1, \ldots, k$.

In Section 5 we will show, in one specific example, how to apply our results to bound effective moduli. The goal is to minimize the amount of linear algebra and optimization to be made and perform it once and for all. The results of the present section will turn out to be very useful in that respect. The basic fact is that in the 
kind of applications we shall discuss in Section 5 it is particulary useful to know, for a given jointly rank- $r$ convex, invariant 2-form $F$, the vector space $N_{F}$ defined in Definition 2.12, because, as we shall see in Section 5 , only in those subspaces one can obtain information which is not already recoverable by convex forms.

We have shown in the present Section that for extremal forms, if $r \geq 2$ such subspaces have dimension at most $k$, reducing the complexity of the general problem. For instance, for $k$ properties in dimension $d$ one has to deal in principle with matrices of dimension $k d^{2} \times k d^{2}$. We reduce the problem to a problem involving matrices of dimension $k^{2}$. For dimension $d=3$, with $k=2$ the algorithm reduces from 324 to 4 parameters. The price we payed is that we disregard non invariant forms.

\section{BOUNDS IN HOMOGENIZATION}

In this section we shall apply our results to a significant example in the theory of composites. Let us start with a very short review. Let $\mathbb{T}=(0,1)^{3}$. A symmetric $3 \times 3$ matrix valued measurable function $\Sigma$ defined on $\mathbb{R}^{3}$ and $\mathbb{T}$ periodic is called a conductivity matrix if there exists $K>1$ such that

$$
K^{-1} \operatorname{Id}(3) \leq \Sigma \leq K \operatorname{Kd}(3) \quad \text { a.e. in } \mathbb{R}^{3} .
$$

The effective conductivity $\Sigma^{*}$ associated to $\Sigma$ is defined as the unique symmetric $3 \times 3$ matrix satisfying the following properties: $\forall F \in \mathcal{M}(3,3)$

$$
\operatorname{Tr}\left(F \Sigma^{*} F^{t}\right):=\inf _{U \in W_{\sharp}^{1,2}\left(\mathbb{T}, \mathbb{R}^{3}\right)} \int_{\mathbb{T}} \operatorname{Tr}\left((D U+F) \Sigma(x)(D U+F)^{t}\right) \mathrm{d} x .
$$

The space $W_{\sharp}^{1,2}$ denotes as usual the space of zero mean vector fields which are in $W_{\text {loc }}^{1,2}\left(\mathbb{R}^{3}, \mathbb{R}^{3}\right)$ and are $\mathbb{T}$-periodic, see [14].

Remark 5.1. The periodicity assumption is made to keep things as simple as possible. For the general definition through the theory of $G$-convergence and of $H$-convergence (or even $\Gamma$-convergence) the reader is referred to $[14,15,27,44,45,53,58]$.

For a general introduction to the field of bounds see also $[38,61,65]$. To state the general bounds in the present setting we will use the following definition, in which we identify a pair of $d \times d$ matrices with a vector of $2 d^{2}$ components, obtained by juxtaposition of the rows of the first with those of the second matrix.

Definition 5.2. Given a 2-form $F$ on $\mathcal{M}^{2}(d, d) \times \mathcal{M}^{2}(d, d)$, we write $M^{F}$ for the symmetric $2 d^{2} \times 2 d^{2}$ real matrix which satisfies

$$
\left\langle M^{F}\left(\begin{array}{c}
A \\
B
\end{array}\right),\left(\begin{array}{c}
A \\
B
\end{array}\right)\right\rangle:=F(A, B)
$$

for all $A, B$ in $\mathcal{M}(d, d)$.

Note that $M^{F}$ is the Hessian of $F$ divided by 2 . In what follows $\Sigma$ and $\Lambda$ are conductivity matrices in the sense of (5.1).

Theorem 5.3. Let $F$ be a 2 -form and let $M^{F}$ be associated to $F$ via Definition 5.2.

Part I. If $F$ is jointly rank-1 convex, then the inequality

$$
\left(\begin{array}{cc}
\Sigma(x) \otimes I d(3) & 0 \\
0 & \Lambda(x) \otimes I d(3)
\end{array}\right)-M^{F} \geq 0 \quad \text { a.e. in } \mathbb{R}^{3}
$$

implies the inequality

$$
\left(\begin{array}{cc}
\Sigma^{*} \otimes I d(3) & 0 \\
0 & \Lambda^{*} \otimes I d(3)
\end{array}\right) \geq M^{F}+\left(\int_{\mathbb{T}}\left(\left(\begin{array}{cc}
\Sigma(x) \otimes I d(3) & 0 \\
0 & \Lambda(x) \otimes I d(3)
\end{array}\right)-M^{F}\right)^{-1} \mathrm{~d} x\right)^{-1} .
$$


Part II. If $F$ is jointly rank- $(d-1)$ convex, then the inequality

$$
\left(\begin{array}{cc}
\Sigma^{-1}(x) \otimes I d(3) & 0 \\
0 & \Lambda(x)^{-1} \otimes I d(3)
\end{array}\right)-M^{F}>0 \quad \text { a.e. in } \mathbb{R}^{3}
$$

implies the inequality

$$
\left.\left(\begin{array}{cc}
\left(\Sigma^{*}\right)^{-1} \otimes I d(3) & 0 \\
0 & \left(\Lambda^{*}\right)^{-1} \otimes I d(3)
\end{array}\right) \geq M^{F}+\left(\int_{\mathbb{T}}\left(\begin{array}{cc}
\Sigma^{-1}(x) \otimes I d(3) & 0 \\
0 & \Lambda^{-1}(x) \otimes I d(3)
\end{array}\right)-M^{F}\right)^{-1} \mathrm{~d} x\right)^{-1}
$$

The proof is a well known consequence of compensated compactness. We refer to [12], [36] and [58].

From now on we will make the following assumptions.

$$
\begin{gathered}
\Sigma(x)=\left(\sigma_{1} \chi(x)+\sigma_{2}(1-\chi(x)) \operatorname{Id}(3),\right. \\
\Lambda(x)=\left(\lambda_{1} \chi(x)+\lambda_{2}(1-\chi(x)) \operatorname{Id}(3),\right. \\
\Sigma^{*}=\sigma^{*} I d(3), \quad \Lambda^{*}=\lambda^{*} I d(3)
\end{gathered}
$$

and we will set

$$
\theta=\int_{\mathbb{T}} \chi(x) \mathrm{d} x
$$

In physical terms we are assuming that both the first and the second physical properties are locally isotropic and that they have isotropic effective moduli. The function $\chi$ is the characteristic function of some measurable subset of $\mathbb{T}$. The number $\theta$ is called the volume fraction and it obviously satisfies $\theta \in[0,1]$. The next assumption is that we will restrict the forms to be rotationally invariant. In this case, the splitting (2.7) induces a splitting of the matrix $M^{F}=M_{1}^{F}+M_{2}^{F}+M_{3}^{F}$ so that each of the inequalities (5.3), (5.4), (5.5), (5.6) can be written as a system of three inequalities involving lower dimensional matrices. Actually, each of the matrices $M_{i}^{F}$ can be written as a direct sum of two dimensional blocks depending only on $i$, which we continue to call $M_{i}^{F}$ and which, for $i=1,2,3$, have multiplicities $1, \frac{d(d+1)}{2}-1$ and $\frac{d(d-1)}{2}$ respectively.

We now focus on the upper bound. For the lower bound the analysis is very similar, hence it will be omitted. Part II of Theorem 5.3 becomes

$$
\left\{\begin{array}{l}
\left(\begin{array}{cc}
\sigma^{-1}(x) & 0 \\
0 & \lambda^{-1}(x)
\end{array}\right) \geq M_{i}^{F} \quad i=1,2,3 \quad \text { a.e. in } \mathbb{R}^{3} \\
\text { implies } \\
\left(\begin{array}{cc}
\sigma_{*}^{-1} & 0 \\
0 & \lambda_{*}^{-1}
\end{array}\right) \geq M_{i}^{F}+\left(\int_{\mathbb{T}}\left(\left(\begin{array}{cc}
\sigma^{-1}(x) & 0 \\
0 & \lambda^{-1}(x)
\end{array}\right)-M_{i}^{F}\right)^{-1} \mathrm{~d} x\right)^{-1} i=1,2,3 .
\end{array}\right.
$$

Moreover, by construction, the matrices $M_{i}^{F}$ are given by

$$
\begin{aligned}
& M_{1}^{F}=A+B-3 C \\
& M_{2}^{F}=A+B \\
& M_{3}^{F}=A-B
\end{aligned}
$$

where $(A, B, C)$ is the triple associated to $F$ via Definition 2.3. 
Remark 5.4. The same happens if $k>2$. The invariant forms always give rise to three algebraic problems on symmetric elements of $\mathcal{M}(k, k)$. The next step is to show that among invariant two forms attention can be restricted to $(r, d)$ extremal ones. This crucial observation is due to Milton [36] and it has also been exploited by Cherkaev and Gibiansky [20].

We will use the notation

$$
f_{\Omega}:=\frac{1}{\operatorname{vol}(\Omega)} \int_{\Omega}
$$

Lemma 5.5 (Milton). Let $\Omega$ be a bounded measurable domain of $\mathbb{R}^{d}$. Let $\alpha>0$, let $\Psi$ be a symmetric real matrix valued function $\Psi: \Omega \rightarrow \mathcal{M}_{S}(d, d)$ such that $\Psi(x) \geq \alpha I$ a.e. in $\Omega$. $\left(\mathcal{M}_{S}(d, d)\right.$ denotes the vector space of symmetric real valued $d \times d$ matrices). Set $V$ to be the set of symmetric non negative definite matrices $H$ which satisfy $H<\Psi(x)$ a.e. in $\Omega$. and set

$$
\mathcal{T}: V \rightarrow \mathcal{M}_{S}(d, d)
$$

to be

Then

$$
\mathcal{T}(H):=\left(f_{\Omega}(\Psi(x)-H)^{-1} \mathrm{~d} x\right)^{-1}+H
$$

$$
\mathcal{T}(H) \leq \mathcal{T}(0)
$$

for all $H \in V$. Moreover, if $\Psi$ is not constant, $\mathcal{T}(H)<\mathcal{T}(0)$ for all $H \in V \backslash\{0\}$.

Proof. A version of this result is proved in [38]. We give here a self contained alternative proof which maybe of independent interest.

Step 1. For $H \in V$ set

$$
g_{H}:[0,1] \rightarrow \mathcal{M}_{S}(d, d)
$$

to be

$$
g_{H}(t):=\mathcal{T}(t H)
$$

and note that $g_{H}$ is well defined. Indeed if $H \in V$, then $t H \in V$ for all $t \in[0,1]$, as it is easily verified. We want to prove that $g_{H}(1) \leq g_{H}(0)$ for all $H \in V$ and $g_{H}(1)<g_{H}(0)$ if $H \neq 0$ and $\Psi$ is not constant.

We have

$$
g_{H}(1)-g_{H}(0)=\int_{0}^{1} \dot{g}_{H}(s) \mathrm{d} s,
$$

where $\dot{g}_{H}(s)$ denotes differentiation of $g_{H}(s)$ with respect to $s$.

Claim 1. Let $H \in V, H \neq 0, t \in(0,1)$. Then

$\dot{g_{H}}(t)=H-\left(f_{\Omega}(\Psi(x)-t H)^{-1} \mathrm{~d} x\right)^{-1} \cdot f_{\Omega}(\Psi(x)-t H)^{-1} H(\Psi(x)-t H)^{-1} \mathrm{~d} x \cdot f_{\Omega}(\Psi(x)-t H)^{-1} \mathrm{~d} x$.

Claim 2. For all $H \in V$ and for all $t \in(0,1)$ we have $\dot{g}_{H}(t) \leq 0$. Moreover, if $H \in V \backslash\{0\}$ and $\Psi$ is not constant, then $\dot{g}_{H}(t)<0$.

Assuming Claims 1 and 2, and using (5.11) the proof of Lemma 5.5 is concluded. It remains to prove Claims 1 and 2 .

Proof of Claim 1. For any $A:(0,1) \rightarrow \mathcal{M}_{S}(d, d)$ of class $C^{1}$ we have the well known formula (see [9])

$$
\frac{\mathrm{d}}{\mathrm{d} t} A^{-1}(t)=-A^{-1}(t) \dot{A}(t) A^{-1}(t) .
$$


Using (5.13) one has

$\dot{g}_{H}(t)=H-\left(f_{\Omega}(\Psi(x)-t H)^{-1} \mathrm{~d} x\right)^{-1} \cdot\left(\frac{\mathrm{d}}{\mathrm{d} t} f_{\Omega}(\Psi(x)-t H)^{-1} \mathrm{~d} x\right)^{-1} \cdot\left(f_{\Omega}(\Psi(x)-t H)^{-1} \mathrm{~d} x\right)^{-1}$.

By using (5.13) again we get Claim 1.

Proof of Claim 2. By (5.12) the statement is equivalent to

$$
\left(f_{\Omega}(\Psi(x)-t H)^{-1} \mathrm{~d} x\right) H\left(f_{\Omega}(\Psi(x)-t H)^{-1} \mathrm{~d} x\right) \leq f_{\Omega}\left((\Psi(x)-t H)^{-1} H(\Psi(x)-t H)^{-1}\right) \mathrm{d} x .
$$

Set

Then (5.15) can be written as

$$
B(x):=H^{1 / 2}(\Psi(x)-t H)^{-1} .
$$

$$
f_{\Omega} B^{t}(x) B(x) \mathrm{d} x \geq\left(f_{\Omega} B^{t}(x) \mathrm{d} x\right)\left(f_{\Omega} B(x) \mathrm{d} x\right) .
$$

Note that the inequality $M^{t} M \geq 0$ for all matrices $M \in \mathcal{M}(d, d)$ implies

$$
\left(B^{t}(x)-f_{\Omega} B^{t}(x) \mathrm{d} x\right)\left(B(x)-f_{\Omega} B(x) \mathrm{d} x\right) \geq 0 \quad \text { a.e. in } \Omega
$$

and the latter implies

which is equivalent to

$$
f_{\Omega}\left(B^{t}(x)-f_{\Omega} B^{t}(x) \mathrm{d} x\right)\left(B(x)-f_{\Omega} B(x) \mathrm{d} x\right) \mathrm{d} x \geq 0
$$

$$
f_{\Omega} B^{t}(x) B(x) \mathrm{d} x \geq\left(f_{\Omega} B^{t}(x) \mathrm{d} x\right)\left(f_{\Omega} B(x) \mathrm{d} x\right) .
$$

This proves that (5.15) holds. Moreover equality in (5.15) holds if and only if it holds in (5.17) i.e. if and only if

$$
B(x)=f_{\Omega} B(x) \mathrm{d} x .
$$

By (5.16) the latter is equivalent to the fact that $\Psi$ is constant.

In order to simplify notations we introduce the two by two matrices

$$
\Psi(x):=\left(\begin{array}{cc}
\sigma^{-1}(x) & 0 \\
0 & \lambda^{-1}(x)
\end{array}\right)
$$

and

$$
\Psi^{*}:=\left(\begin{array}{cc}
\sigma_{*}^{-1} & 0 \\
0 & \lambda_{*}^{-1}
\end{array}\right) .
$$

By Lemma 3.1, $M_{2}^{F}$ and $M_{3}^{F}$ as defined in (5.8) are nonnegative. Therefore, by Lemma 5.5, $(5.7)_{2}$ and $(5.7)_{3}$ can, at the very best, deliver the bound

$$
\Psi^{*} \geq\left(\int_{\mathbb{T}} \Psi(x)^{-1} \mathrm{~d} x\right)^{-1}
$$

i.e. the well known "arithmetic mean" bound. 
Moreover (5.7) ${ }_{1}$ can deliver a better bound than (5.20) only if $A+B-3 C$ is not positive definite.

When $F$ is extremal, $F$ is associated to $\left(\frac{r}{2} H, \frac{r}{2} H, H\right)$. Since $d=3$ and $r=2$, we have that $F$ is associated to $(H, H, H)$ and therefore

where $H$ is a nonnegative definite symmetric matrix:

$$
\left\{\begin{array}{lll}
M_{1}^{F} & = & -H \\
M_{2}^{F} & = & 2 H \\
M_{3}^{F} & = & 0
\end{array}\right.
$$

$$
H=\left(\begin{array}{ll}
h_{11} & h_{12} \\
h_{12} & h_{22}
\end{array}\right) \geq 0
$$

After some easy manipulations (5.7) becomes:

$$
\left\{\begin{array}{ll}
\Psi(x)-2 H & \geq 0 \\
H & \geq 0
\end{array} \quad \text { a.e. in } \mathbb{R}^{3}\right.
$$

implies

$$
\left\{\begin{array}{l}
(5.20) \\
\Psi^{*} \geq-H+\left(\int_{\mathbb{T}}(\Psi(x)+H)^{-1} \mathrm{~d} x\right)^{-1} \\
\Psi^{*} \geq 2 H+\left(\int_{\mathbb{T}}(\Psi(x)-2 H)^{-1} \mathrm{~d} x\right)^{-1} .
\end{array}\right.
$$

Using (5.9) with the choice (5.18), conditions (5.22) can be rewritten in the following fashion:

$$
\Psi^{*} \geq\left\{\begin{array}{c}
\mathcal{T}(0) \\
\mathcal{T}(-H) \\
\mathcal{T}(2 H)
\end{array}\right.
$$

Remark 5.6. Lemma 5.5 implies that, given two symmetric nonnegative matrices $H_{1}$ and $H_{2}$

$$
\text { if } H_{1} \geq H_{2} \text {, then } \mathcal{T}\left(H_{1}\right) \leq \mathcal{T}\left(H_{2}\right) \text {. }
$$

Using Remark 5.6 we obtain that (5.23) is equivalent to $\Psi^{*} \geq \mathcal{T}(-H)$.

Now, for simplicity, we make some further assumptions leading to the so-called interchange inequality problem. We assume that

$$
\left\{\begin{array}{l}
\sigma_{1}=\frac{1}{K}=\lambda_{2} \\
\sigma_{2}=K=\lambda_{1}
\end{array}\right.
$$

and we set

$$
D_{1}=\left(\begin{array}{cc}
\frac{1}{K} & 0 \\
0 & K
\end{array}\right) \quad D_{2}=\left(\begin{array}{cc}
K & 0 \\
0 & \frac{1}{K}
\end{array}\right) \text {. }
$$

There are still three parameters to optimize over, the entries of the matrix $H$. We obtain:

$$
(5.21) \Rightarrow(5.22)
$$

if and only if

$$
\left\{\begin{array}{ll}
D_{1}-2 H & \geq 0 \\
D_{2}-2 H & \geq 0 \\
H & \geq 0
\end{array} \Rightarrow \Psi^{*} \geq \mathcal{T}(-H)\right.
$$


One can check, using a monotonicity argument of the same type of Lemma 5.5 that we omit, that the best bound in (5.27) is obtained when $H$ satisfies

$$
\operatorname{det}\left(D_{1}-2 H\right)=\operatorname{det}\left(D_{2}-2 H\right)=0 \text {. }
$$

The corresponding matrices $H$ depend only on one parameter, called $\alpha$ and one easily checks that

$$
H(\alpha)=\left(\begin{array}{cc}
\alpha & \beta^{ \pm}(\alpha) \\
\beta^{ \pm}(\alpha) & \alpha
\end{array}\right)
$$

with $\beta^{ \pm}(\alpha):= \pm \sqrt{(K-\alpha)\left(\frac{1}{K}-\alpha\right)}$ and $\alpha \in I:=\left[\frac{1}{K}, \frac{K}{K^{2}+1}\right]$. Finally, it is easy to check that

$$
H_{0}:=H\left(\frac{K}{K^{2}+1}\right) \geq H(\alpha), \quad \forall \alpha \in I .
$$

Hence, by Remark 5.6, the best bound in (5.27) is obtained for

$$
H=H_{0}=\frac{K}{K^{2}+1}\left(\begin{array}{ll}
1 & 1 \\
1 & 1
\end{array}\right) .
$$

The above choice $H=H_{0}$ delivers the following upper bound:

$$
6 \sigma^{*} \lambda^{*}+\frac{7 K^{2}+5}{K\left(1+K^{2}\right)}\left(\sigma^{*}+\lambda^{*}\right)+K^{2}+4+\frac{1}{K^{2}} \leq 0 .
$$

We recall that the already known lower bound has the following explicit expression:

$$
\left(K+\frac{1}{K}\right)\left(\sigma^{*} \lambda^{*}-2\right)+\sigma^{*}+\lambda^{*} \geq 0 .
$$

We set $L_{K}=\frac{2+4 K^{2}}{5 K+K^{3}}$ and $U_{K}=\frac{2 K\left(2+K^{2}\right)}{1+5 K^{2}}$. They represent the lower and upper Hashin-Shtrikman bounds for a mixture of two isotropic phases with conductivities $K$ and $\frac{1}{K}$ and volume fractions $\frac{1}{2}$ and $\frac{1}{2}$.

In Figure 1 we display simultaneously (5.31) and (5.32) for $K=9$. It is well known that the upper bound is attained at five points, which are spotted in Figure 2 for the case $K=9$. For the derivation of the latter result the reader is referred to $[34,35,52]$. The pertinent microgeometries use an idea of Schulgasser and were found by Milton. They are described in Section 11 of [5]. We will not digress further on this. Let us just point out that quite a number of authors have given contributions to the subject including Beran [6], Bergman [7], Milton [34,35] and, Allaire and Maillot [4]. In two dimension we mention the work of Keller [28] and Dykhne [17]. For a more general situation when (5.25) does not hold and the effective conductivities are not necessarily isotropic see Gibiansky and Cherkaev [12], which fully characterize the range of the pair of matrices $\Sigma_{*}$ and $\Lambda_{*}$ completing previous work of Milton [34,35], considering only isotropic composites. See also Clark and Milton [13]. For phase interchange inequalities, see [5] and, for more than two phases the reader is referred to $[47,65]$. In the latter works, the authors, in particular, gave a new proof of the lower bound used in the present section. The previous derivation, in [5], was slightly incorrect.

Remark 5.7. The way we optimized the family of bounds (5.27) over the admissible matrices $H$ is essentially to use Lemma 5.5. The result is that one can choose $H$ so that $\operatorname{rank}\left(D_{1}-2 H\right)=\operatorname{rank}\left(D_{2}-2 H\right)=1$.

We learned the idea of trying to maximize the number $\operatorname{dim}\left(\operatorname{Ker}\left(D_{1}-2 H\right)\right)+\operatorname{dim}\left(\operatorname{Ker}\left(D_{2}-2 H\right)\right)$ from $[12]$ (see also $[11,38])$.

The suggestion of Cherkaev-Gibiansky is very intuitive because the largest the kernels of $D_{1}-2 H, D_{2}-2 H$ is, the weaker the conditions that a putative optimal field should satisfy are. As it turns out, one needs some extra considerations to find the optimal $H$. 


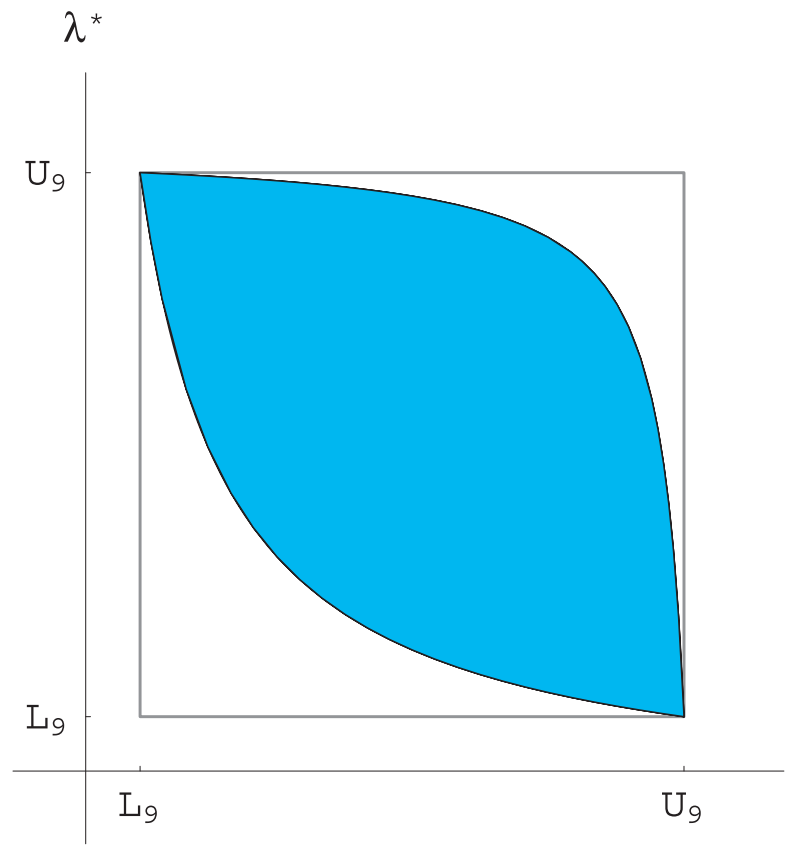

Figure 1. Region bounded by best available bounds for $K=9$.

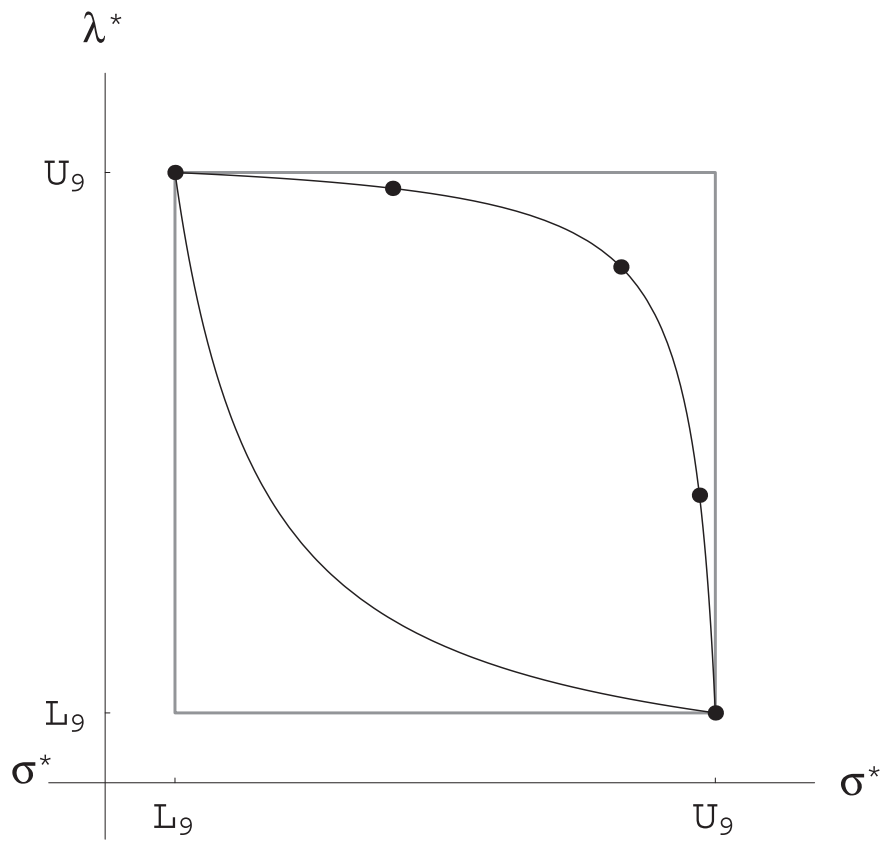

Figure 2. The "Milton-Schulgasser" points, $K=9$.

Remark 5.8. Optimality conditions are a very important issue. Let us point out that the results of Section 4 provide a basis for a systematic study. For instance, an optimal field must have constant antisymmetric part (zero if such is the average field).

Remark 5.9. The result of the present section can be extended to deliver bounds for anisotropic composites in contrast to the results using complex variable techniques. Extension to more than two physical properties is also possible.

\section{The "MIXED" CASE}

The theory developed so far is not yet general enough to treat many interesting cases falling, in a natural way, in the framework of compensated compactness. In particular, we would like to consider a framework general enough to include the well known div-curl lemma of Murat and Tartar. In the latter case one considers scalar products of sequences satisfying (1.7) and (1.8) with sequences satisfying (1.10) and (1.11).

To make the exposition clear we begin with the case $k=2$ and restrict attention, as usual to invariant 2 -forms. Throughout this section we consider only the case $d \geq 3$. We look for jointly rank- $(1,(d-1))$ convex forms in the following sense.

Definition 6.1. A 2 -form $F$ on $\mathcal{M}^{2}(d, d)$ is rank- $(r, d-r)$ convex if for any pair of $d \times d$ matrices $(P, Q)$ such that

$$
\begin{aligned}
& \operatorname{dim}(\operatorname{KerP})=d-r, \\
& \operatorname{dim}(\operatorname{KerQ})=r \text {, } \\
& \operatorname{Ker} P \text { and } \operatorname{Ker} Q \quad \text { are orthogonal }
\end{aligned}
$$

one has

$$
F(P, Q) \geq 0 .
$$


Remark 6.2. For a rank- $(r, d-r)$ convex 2-form $F$, the positivity of $F(P, Q)$ is automatically guaranteed for a slightly more general class of matrices, using the following result.

Lemma 6.3. Let $F$ be a rank- $(r, d-r)$ convex 2-form. Let $\mathcal{K}$ and $\mathcal{L}$ be two orthogonal subspaces of $\mathcal{M}^{2}(d, d)$ such that $\operatorname{dim}(\mathcal{K})=d-r$ and $\operatorname{dim}(\mathcal{L})=r$ and let $P, Q \in \mathcal{M}^{2}(d, d)$ be two matrices such that $\operatorname{Ker} P \supseteq \mathcal{K}$ and $\operatorname{Ker} Q \supseteq \mathcal{L}$. Then $F(P, Q) \geq 0$.

Proof. A simple continuity argument that we omit.

Definition 6.1 is the right one to apply the compensated compactness theory. The div-curl lemma of Murat and Tartar considers the case $r=1$ :

Theorem 6.4 (Tartar and Murat). Let $\Omega$ be an open bounded subset of $\mathbb{R}^{d}$ and let $\left(P_{\epsilon}, Q_{\epsilon}\right),\left(P_{0}, Q_{0}\right)$ be in $L^{2}\left(\Omega ; \mathbb{R}^{d \times d}\right)$ and satisfying (1.7), (1.8), (1.10) and (1.11).

Let $F$ be a jointly rank-(1,d-1) convex 2 -form then, for any $\phi \geq 0$ with $\phi \in C_{c}(\Omega)$, we have

$$
\liminf _{\epsilon \rightarrow 0^{+}} \int_{\Omega} \phi F\left(P_{\epsilon}, Q_{\epsilon}\right) \mathrm{d} x \geq \int_{\Omega} \phi F\left(P_{0}, Q_{0}\right) \mathrm{d} x .
$$

The div-curl lemma corresponds to the choice $F(P, Q)=\operatorname{Tr}\left(P Q^{t}\right)$. As it is well known $\operatorname{Tr}\left(P Q^{t}\right)=0$ for all $P$ and $Q$ satisfying (6.1), (6.2) and (6.3). Therefore, for $F(P, Q)=\operatorname{Tr}\left(P Q^{t}\right)$ equality holds in (6.5).

In this section we prove two results about invariant jointly rank- $(1,(d-1))$ convex 2 -forms. The first gives the analogue of Lemma 3.1. First we need a further definition.

Definition 6.5. Given positive integers, $r, k_{1}$ and $k_{2}$, set $k=k_{1}+k_{2}$. A 2-form $F$ on $\mathcal{M}^{k}(d, d)$ is $\left(k_{1}, k_{2}\right)$-jointly rank- $(r, d-r)$ convex $(1 \leq r<d)$ if for any $\left(P^{1}, \ldots, P^{k_{1}}\right) \in \mathcal{M}^{k_{1}}(d, d)$ and any $\left(Q^{1}, \ldots, Q^{k_{2}}\right) \in \mathcal{M}^{k_{2}}(d, d)$ such that

$$
\begin{gathered}
U=\operatorname{Ker} P^{1}=\cdots=\operatorname{Ker} P^{k_{1}}, \quad \operatorname{dim}(\operatorname{Ker}(U))=d-r, \\
W=\operatorname{Ker} Q^{1}=\cdots=\operatorname{Ker} Q^{k_{2}}, \quad \operatorname{dim}(\operatorname{Ker}(W))=r, \\
U, \quad W \quad \text { orthogonal }
\end{gathered}
$$

one has

$$
F\left(P^{1}, \ldots, P^{k_{1}}, Q^{1}, \ldots, Q^{k_{2}}\right) \geq 0 .
$$

As in Lemma 6.3 the positivity of $F\left(P^{1}, \ldots, P^{k_{1}}, Q^{1}, \ldots, Q^{k_{2}}\right)$ is automatically guaranteed for a slightly more general class of matrices.

Lemma 6.6. Let $r, k_{1}, k_{2}$ be positive integers, and let $k=k_{1}+k_{2}$. Let $F$ be a 2 -form which is $\left(k_{1}, k_{2}\right)$-jointly rank- $(r, d-r)$ convex $(1 \leq r<d)$. Let $\left(P^{1}, \ldots, P^{k_{1}}\right) \in \mathcal{M}^{k_{1}}(d, d)$ and $\left(Q^{1}, \ldots, Q^{k_{2}}\right) \in \mathcal{M}^{k_{2}}(d, d)$. Let $\mathcal{K}$ and $\mathcal{L}$ be two orthogonal subspaces of $\mathcal{M}^{2}(d, d)$ for which $\operatorname{dim}(\mathcal{K})=d-r$, $\operatorname{dim}(\mathcal{L})=r$ and such that

$$
\mathcal{K} \subseteq \bigcap_{i=1}^{k_{1}} \operatorname{Ker} P^{i}, \quad \mathcal{L} \subseteq \bigcap_{j=1}^{k_{2}} \operatorname{Ker} Q^{j} .
$$

Then

$$
F\left(P^{1}, \ldots, P^{k_{1}}, Q^{1}, \ldots, Q^{k_{2}}\right) \geq 0 .
$$

Proof. As for Lemma 6.3, a simple continuity argument that we omit.

Lemma 6.7. Let $d \geq 3, k_{1}, k_{2}$ be two positive integers and let $F: \mathcal{M}^{k_{1}+k_{2}}(d, d) \rightarrow \mathbb{R}$ be an invariant 2 -form associated to $(A, B, C)$ written in $\left[k_{1}, k_{2}\right]$ block form as follows (see (2.22))

$$
A=\left(\begin{array}{cc}
\hat{A} & A_{E} \\
A_{E}^{t} & \tilde{A}
\end{array}\right)
$$


where $\hat{A} \in \mathcal{M}\left(k_{1}, k_{1}\right), A_{E} \in \mathcal{M}\left(k_{1}, k_{2}\right), \tilde{A} \in \mathcal{M}\left(k_{2}, k_{2}\right)$, and similarly for $B$ and $C$. Then $F$ is $\left(k_{1}, k_{2}\right)$ jointly rank- $(1, d-1)$ convex if and only if

$$
\begin{gathered}
\tilde{A}+\tilde{B} \geq 0 \\
\tilde{A}-\tilde{B} \geq 0 \\
\left(\begin{array}{cc}
\hat{A} & B_{E} \\
B_{E}^{t} & \tilde{A}
\end{array}\right) \geq 0 \\
\left(\begin{array}{cc}
\hat{A}+\hat{B} & 0 \\
0 & \frac{\tilde{A}+\tilde{B}}{d-1}
\end{array}\right)-C \geq 0 .
\end{gathered}
$$

Proof. Using the arguments of Section 2, the $O(d)$-invariance of $F$ implies that it is enough to check positivity of the restricted form $F \Downarrow$. In this case, this means to consider $F\left(P^{1}, \ldots, P^{k_{1}}, Q^{1}, \ldots, Q^{k_{2}}\right)$ for matrices of the form

$$
P^{i}=\left(\begin{array}{c|c}
n^{i} & v_{P^{i}} \\
\hline 0 & 0
\end{array}\right) \quad Q^{j}=\left(\begin{array}{c|c}
0 & 0 \\
\hline v_{Q^{j}} & N^{j}
\end{array}\right)
$$

where $n^{i} \in \mathcal{M}(1,1), v_{P^{i}} \in \mathcal{M}(1,(d-1)), v_{Q^{j}} \in \mathcal{M}((d-1), 1)$ and $N^{j} \in \mathcal{M}((d-1),(d-1))$. The restriction of $F$ to matrices of the above form is denoted as usual by

$$
F \Downarrow=F \Downarrow\left(n_{1}, \ldots, n_{k}, v_{P^{1}}, \ldots, v_{P^{k_{1}}}, v_{Q^{1}}, \ldots, v_{Q^{k_{2}}}, N^{1}, \ldots, N^{k_{2}}\right)
$$

We use the following elementary identities

$$
\begin{aligned}
\operatorname{Tr}\left(P^{i}\left(P^{j}\right)^{t}\right) & =n_{i} n_{j}+\left\langle v_{P^{i}}, v_{P^{j}}\right\rangle \\
\operatorname{Tr}\left(Q^{i}\left(Q^{j}\right)^{t}\right) & =\operatorname{Tr}\left(N^{i}\left(N^{j}\right)^{t}\right)+\left\langle v_{Q^{i}}, v_{Q^{j}}\right\rangle \\
\operatorname{Tr}\left(P^{i}\left(Q^{j}\right)^{t}\right) & =0 \\
\operatorname{Tr}\left(P^{i} P^{j}\right) & =n_{i} n_{j} \\
\operatorname{Tr}\left(Q^{i} Q^{j}\right) & =\operatorname{Tr}\left(N^{i} N^{j}\right) \\
\operatorname{Tr}\left(P^{i} Q^{j}\right) & =\left\langle v_{P^{i}}, v_{Q^{j}}\right\rangle \\
\operatorname{Tr}\left(P^{i}\right) \operatorname{Tr}\left(P^{j}\right) & =n_{i} n_{j} \\
\operatorname{Tr}\left(Q^{i}\right) \operatorname{Tr}\left(Q^{j}\right) & =\operatorname{Tr}\left(N^{i}\right) \operatorname{Tr}\left(N^{j}\right) \\
\operatorname{Tr}\left(P^{i}\right) \operatorname{Tr}\left(Q^{j}\right) & =n_{i} \operatorname{Tr}\left(N^{j}\right) .
\end{aligned}
$$

Moreover, since $d \geq 3$, we have

$$
\begin{aligned}
\operatorname{Tr}\left(N^{i}\left(N^{j}\right)^{t}\right) & =\sum_{k=1}^{3}\left\langle\mathbb{P}_{k} N^{i}, \mathbb{P}_{k} N^{j}\right\rangle \\
\operatorname{Tr}\left(N^{i} N^{j}\right) & =\sum_{k=1}^{2}\left\langle\mathbb{P}_{k} N^{i}, \mathbb{P}_{k} N^{j}\right\rangle-\left\langle\mathbb{P}_{3} N^{i}, \mathbb{P}_{3} N^{j}\right\rangle \\
\operatorname{Tr}\left(N^{i}\right) \operatorname{Tr}\left(N^{j}\right) & =(d-1)\left\langle\mathbb{P}_{1} N^{i}, \mathbb{P}_{1} N^{j}\right\rangle .
\end{aligned}
$$

We claim that writing $F \Downarrow$ with the help of (6.16)-(6.27) and using the projections $\mathbb{P}_{i}$ one obtains the assertion. We now check our claim. We write $\hat{a}_{i j}, \tilde{a}_{i j}$ and $a_{i j}^{E}$ for the entries of $\hat{A}, \tilde{A}, A^{E}$ respectively. We do the same for 
$B$ and $C$. We have

$$
\begin{aligned}
F \Downarrow= & \sum_{i, j=1}^{k_{1}}\left[\hat{a}_{i j} \operatorname{Tr}\left(P^{i}\left(P^{j}\right)^{t}\right)+\hat{b}_{i j} \operatorname{Tr}\left(P^{i} P^{j}\right)-\hat{c}_{i j} \operatorname{Tr}\left(P^{i}\right) \operatorname{Tr}\left(P^{j}\right)\right] \\
& +\sum_{i, j=1}^{k_{2}}\left[\tilde{a}_{i j} \operatorname{Tr}\left(Q^{i}\left(Q^{j}\right)^{t}\right)+\tilde{b}_{i j} \operatorname{Tr}\left(Q^{i} Q^{j}\right)-\tilde{c}_{i j} \operatorname{Tr}\left(Q^{i}\right) \operatorname{Tr}\left(Q^{j}\right)\right] \\
& +2 \sum_{i=1}^{k_{1}} \sum_{j=1}^{k_{2}}\left[a_{i j}^{E} \operatorname{Tr}\left(P^{i}\left(Q^{j}\right)^{t}\right)+b_{i j}^{E} \operatorname{Tr}\left(P^{i} Q^{j}\right)-c_{i j}^{E} \operatorname{Tr}\left(P^{i}\right) \operatorname{Tr}\left(Q^{j}\right)\right] .
\end{aligned}
$$

By using equations (6.16)-(6.27) it is easy to verify that the above expression can be rewritten as

$$
\begin{aligned}
F \Downarrow= & \sum_{i, j=1}^{k_{1}} \hat{a}_{i j}\left\langle v_{P^{i}}, v_{P^{j}}\right\rangle+\sum_{i, j=1}^{k_{2}} \tilde{a}_{i j}\left\langle v_{Q^{i}}, v_{Q^{j}}\right\rangle+2 \sum_{i=1}^{k_{1}} \sum_{j=1}^{k_{2}} b_{i j}^{E}\left\langle v_{P^{i}}, v_{Q^{j}}\right\rangle \\
& +\sum_{i, j=1}^{k_{2}}\left(\tilde{a}_{i j}+\tilde{b}_{i j}\right)\left\langle\mathbb{P}_{2}\left(N^{i}\right), \mathbb{P}_{2}\left(N^{j}\right)\right\rangle+\sum_{i, j=1}^{k_{2}}\left(\tilde{a}_{i j}-\tilde{b}_{i j}\right)\left\langle\mathbb{P}_{3}\left(N^{i}\right), \mathbb{P}_{3}\left(N^{j}\right)\right\rangle \\
& +\sum_{i, j=1}^{k_{1}}\left(\hat{a}_{i j}+\hat{b}_{i j}-\hat{c}_{i j}\right) n_{i} n_{j} \\
& +\sum_{i, j=1}^{k_{2}}\left(\frac{\tilde{a}_{i j}+\tilde{b}_{i j}}{d-1}-\tilde{c}_{i j}\right) \operatorname{Tr}\left(N^{i}\right) \operatorname{Tr}\left(N^{j}\right) \\
& -2 \sum_{i=1}^{k_{1}} \sum_{j=1}^{k_{2}} c_{i j}^{E} n_{i} \operatorname{Tr}\left(N^{j}\right) .
\end{aligned}
$$

The variables $v_{P^{i}}, v_{Q^{j}}, n_{i}, N^{j}$ are all independent from each others. Therefore the latter expression is positive if and only if the following four conditions hold:

$$
\begin{gathered}
\sum_{i, j=1}^{k_{1}} \hat{a}_{i j}\left\langle v_{P^{i}}, v_{P^{j}}\right\rangle+\sum_{i, j=1}^{k_{2}} \tilde{a}_{i j}\left\langle v_{Q^{i}}, v_{Q^{j}}\right\rangle+2 \sum_{i=1}^{k_{1}} \sum_{j=1}^{k_{2}} b_{i j}^{E}\left\langle v_{P^{i}}, v_{Q^{j}}\right\rangle \geq 0, \\
\sum_{i, j=1}^{k_{2}}\left(\tilde{a}_{i j}+\tilde{b}_{i j}\right)\left\langle\mathbb{P}_{2} N^{i}, \mathbb{P}_{2} N^{j}\right\rangle \geq 0, \\
\sum_{i, j=1}^{k_{2}}\left(\tilde{a}_{i j}-\tilde{b}_{i j}\right)\left\langle\mathbb{P}_{3} N_{i}, \mathbb{P}_{3} N_{j}\right\rangle \geq 0, \\
\sum_{i, j=1}^{k_{1}}\left(\hat{a_{i j}}+\hat{b_{i j}}-\hat{c_{i j}}\right) n_{i} n_{j}+\sum_{i, j=1}^{k_{2}}\left(\frac{\tilde{a}_{i j}+\tilde{b}_{i j}}{d-1}-\tilde{c}_{i j}\right) \operatorname{Tr}\left(N^{i}\right) \operatorname{Tr}\left(N^{j}\right)-2 \sum_{i=1}^{k_{1}} \sum_{j=1}^{k_{2}} c_{i j}^{E} n_{i} \operatorname{Tr}\left(N^{j}\right) \geq 0 .
\end{gathered}
$$

Clearly (6.28), (6.29) and (6.30) are equivalent to (6.14), (6.12) and (6.13) respectively and (6.31) is equivalent to

$$
\left(\begin{array}{cc}
\hat{A}+\hat{B}-\hat{C} & -C_{E} \\
-C_{E}^{t} & \frac{\tilde{A}+\tilde{B}}{d-1}-\tilde{C}
\end{array}\right) \geq 0
$$

which is equivalent to $(6.15)$.

The next result characterizes the analogue of quasi-affine functions. We need a definition. 
Definition 6.8. Given positive integers $k_{1}, k_{2}$ set $k=k_{1}+k_{2}$. We say that an invariant 2 -form $\mathrm{F}$ is $\left(k_{1}, k_{2}\right)$ jointly rank- $(1, d-1)$ affine if both $F$ and $-F$ are $\left(k_{1}, k_{2}\right)$-jointly rank- $(1, d-1)$ convex.

Corollary 6.9. An invariant 2 -form $F$ is $\left(k_{1}, k_{2}\right)$-jointly rank- $(1, d-1)$ affine $(d \geq 3)$ if and only if $F$ is associated to

$$
\left(\begin{array}{c|c}
0 & A_{E} \\
\hline A_{E} & 0
\end{array}\right), \quad\left(\begin{array}{c|c}
H & 0 \\
\hline 0 & 0
\end{array}\right), \quad\left(\begin{array}{c|c}
H & 0 \\
\hline 0 & 0
\end{array}\right)
$$

for some symmetric $H \in \mathcal{M}\left(k_{1}, k_{1}\right)$ and some arbitrary $A_{E} \in \mathcal{M}\left(k_{1}, k_{2}\right)$.

Sketch of the proof. We need to choose $A, B, C$ so that the inequalities (6.12)-(6.15) are satisfied as equalities. The first two implies $\tilde{A}=\tilde{B}=0$. The third one implies $\hat{A}=B_{E}=0$. Finally the fourth one implies $\hat{C}=\hat{A}+\hat{B}$ and $\tilde{C}=\frac{\tilde{A}+\tilde{B}}{d-1}$.

Corollary 6.9 may be already known although we were unable to locate the right reference in the literature.

As usual we would like to classify "extremal forms" but we do not have a complete picture yet. This will be left for future work.

\section{Concluding Remarks And open problems}

Our work is tightly linked to the issue of semicontinuity of integral functionals of the kind $I(B)=\int_{\Omega} f(B) \mathrm{d} x$ with $B \in \mathcal{M}(d, d)$. When $B=D U$ and the integrand is not a 2 -form, the notion of rank-one convexity has to be compared with that of quasiconvexity introduced by Morrey [41] as opposed to the classical convexity theory valid for scalar functions and initiated by Tonelli [62]. In this general context the notions of quasiconvexity and rank-one convexity turn out to be different (at least when $d=3$ ) as proved by Šverak in [55]. Similarly, if $B$ satisfies a linear differential constraint of the form $\mathcal{A} B=0$, when $r \geq 2$, rank $r$-convexity must be compared with the appropriate notion of $\mathcal{A}$ quasiconvexity introduced by Fonseca and Muller [18]. Our results have an impact in this more general context. For instance, when considering integral functionals of the form $I(U)=\int_{\Omega} f(D U)$ with $f$ not quasiconvex, one may attempt to compute a lower bound for the quasiconvexification $Q f$ of $f$ by using the same kind of ideas used in composites. If $f$ is continuous and it grows at least quadratically at infinity, the method works. An explicit example can be found in the pioneering work of Kohn and Strang (see [29-31]) and more recently in many papers including [1,3], just to cite a few. In this context one faces exactly the same difficulties explained in the context of homogenization in Section 5. Our results may be valuable in those circumstances.

The issue of finding "extremal", say quasiconvex functions arise explicitly in the work of several authors including Milton [36], Allaire and Kohn [2] and Šverak [54]. The present paper represents a contribution toward that goal: for smooth functions, our work selects the extremal quadratic part, thanks to the theory of compensated compactness of Tartar.

Many questions, even in the quadratic case, remain open. Let us quote a few of them. First, we do not characterize extremal forms for what we called the mixed case. This seems an interesting subject left for some future work. Second, no general results are available for non invariant forms, but it is most likely (see [16,20]) that some of them are "extremal". Their algebraic structure however seems rather intricate. Their characterization would be very useful in applications. Third, finding extremal $S O(n)$ invariant forms requires new techniques. In particular one needs to include forms which are $S O(n)$ invariant but not necessarily $O(n)$ invariant. Such forms exist if $n$ is even and they can be quadratic for $n=2$ and $n=4$. In general $S O(n)$ invariant of matrices are built by adding a further typical invariant (in the sense of Weyl) to the trace, i.e. the complete polarization of the pfaffian (see [51]).

In "practical" terms our results were tested on a difficult problem as explained in Section 5. Our results establish the upper bound for isotropic composites. It is better than those obtained by various authors including the bounds obtained in [4] by using $H$-measures techniques. It coincides with the best known bound which was obtained by different techniques (see $[6,34,35]$ ). Our method has the advantage of showing very clearly how to 
get similar bounds for more than two properties and/or for anisotropic composites. In contrast, the previous derivations in $3 \mathrm{D}$ seem limited to the case of two physical properties and to isotropic composites.

Acknowledgements. The authors thank G.W. Milton and C. Procesi for very fruitful exchange of ideas. V.N. also is grateful to A. Cherkaev, L. Gibiansky and L. Tartar for insightful suggestions and discussions. We are especially grateful to one of the referees who contributed to make this paper a better paper.

E.R. and V.N. acknowledge support from Cofin 40\%. V.N. acknowledges partial support from the University of Utah through Grant AR0 41263/MA and DMS-0411035.

\section{REFERENCES}

[1] G. Allaire and G. Francfort, Existence of minimizers for non-quasiconvex functionals arising in optimal design. Ann. Inst. H. Poincaré Anal. non Linéaire 15 (1998) 301-339.

[2] G. Allaire and R.V. Kohn, Optimal lower bounds on the elastic energy of a composite made from two non-well ordered isotropic materials. Quart. Appl. Math. LII (1994) 311-333.

[3] G. Allaire and V. Lods, Minimizer for a double-well problem with affine boundary conditions. Proc. Roy. Soc. Edinburgh Sec. A 129 (1999) 439-466.

[4] G. Allaire and H. Maillot, $H$-measures and bounds on the effective properties of composite materials. Port. Math. (N.S.) 60 (2003) 161-192.

[5] M. Avellaneda, A.V. Cherkaev, K.A. Lurie and G.W. Milton, On the effective conductivity of polycrystals and a three dimensional phase interchange inequality. J. Appl. Phys. 63 (1988) 4989-5003.

[6] M.J. Beran, Nuovo Cimento 38 (1965) 771-782.

[7] D.J. Bergman, The dielectric constant of a composite material: a problem in classical physics. Phys. Rep. 43 (1978) 377-407.

[8] D.J. Bergman, Rigorous bounds for the complex dielectric constant of a two-component composite. Ann. Physics 138 (1982) $78-114$.

[9] R. Bhatia, Matrix Analysis. Graduate texts in Mathematics, Springer-Verlag, New York (1997).

[10] J.G. Berryman and G.W. Milton, Microgeometry of random composites and porous media. J. Phys. D: Appl. Phys. 21 (1988) $87-94$.

[11] A. Cherkaev, Variational methods for structural optimization. Applied Mathematical Sciences 140, Springer-Verlag, Berlin (2000).

[12] A.V. Cherkaev and L.V. Gibiansky, The exact coupled bounds for effective tensors of electrical and magnetic properties of two-component two-dimensional composites. Proc. Roy. Soc. Edinburgh Sect. A 122 (1992) 93-125.

[13] K. Clark and G. Milton, Optimal bounds correlating electric, magnetic and thermal properties of two phases, two dimensional composites. Proc. R. Soc. Lond. A, 448 (1995) 161-190.

[14] G. Dal Maso, An introduction to $\Gamma$-convergence. Progress in Nonlinear Differential Equations and their Applications 8, Birkhauser Boston, Inc., Boston, MA (1993).

[15] E. De Giorgi and S. Spagnolo, Sulla convergenza degli integrali dell'energia per operatori ellittici del secondo ordine. Bull. Un. Mat. Ital (4) 8 (1973) 391-411.

[16] G. Dell'Antonio and V. Nesi, A scalar inequality which bounds the effective conductivity of composites. Proc. Royal Soc. London A 431 (1990) 519-530.

[17] A.M. Dykhne, Conductivity of a two-dimensional two-phase system. Soviet Physiscs JETP 32 (1971) 63-65.

[18] I. Fonseca and S. Müller, A-quasiconvexity, lower semicontinuity, and Young measures. SIAM J. Math. Anal. 30 (1999) $1355-1390$.

[19] L.V. Gibiansky, Effective properties of a plane two-phase elastic composites: coupled bulk-shear moduli bounds, in Homogenization, Ser. Adv. Math, Appl. Sci. 50, World Sci. Publishing, River Edge, NJ (1999) 214-258.

[20] L.V. Gibiansky and A.V. Cherkaev, Design of composite plates of extremal rigidity and/or Microstructures of composites of extremal rigidity and exact bounds on the associated energy density, in Topics in the mathematical modelling of composite materials, A. Cherkaev and R. Kohn Eds., Progr. Nonlinear Differential Equations Appl. 31, Birkhäuser Boston, Inc., Boston, MA, (1997).

[21] L.V. Gibiansky and A.V. Cherkaev, Coupled estimates for the bulk and shear moduli of a two-dimensional isotropic elastic composite. J. Mech. Phys. Solids 41 (1993) 937-980.

[22] L.V. Gibiansky and S. Torquato, Link between the conductivity and elastic moduli of composite materials. Phys. Rev. Lett. 71 (1993) 2927-2930.

[23] L.V. Gibiansky and S. Torquato, Connection between the conductivity and bulk modulus of Isotropic composite materials. Proc. Roy. Soc. London A 452 (1996) 253-283.

[24] L.V. Gibiansky and S. Torquato, Phase-interchange relations for the elastic moduli of two-phase composites. Internat. J. Engrg. Sci. 34 (1996) 739-760.

[25] G.H. Goldsztein, Rigid-pefectly-plastic two-dimensional polycrystals. Proc. Roy. Soc. Lond. A 457 (2003) 1949-1968. 
[26] Z. Hashin and S. Shtrikman, A variational approach to the theory of effective magnetic permeability of multiphase materials. J. Appl. Phys. 33 (1962) 3125-3131.

[27] V.V. Jikov, S.M. Kozlov and O. A. Oleı̆nik, Homogenization of differential operators and integral functionals. Translated from the Russian by G.A. Yosifian, Springer-Verlag, Berlin (1994).

[28] J.B. Keller, A theorem on the conductivity of a composite medium. J. Math. Phys. 5 (1964) $548-549$.

[29] R.V. Kohn and G. Strang, Optimal design and relaxation of variational problems I. Comm. Pure Appl. Math. 39 (1986) $113-137$

[30] R.V. Kohn and G. Strang, Optimal design and relaxation of variational problems II. Comm. Pure Appl. Math. 39 (1986) 139-182.

[31] R.V. Kohn and G. Strang, Optimal design and relaxation of variational problems III. Comm. Pure Appl. Math. 39 (1986) 353-377.

[32] K.A. Lurie and A.V. Cherkaev, Exact estimates of conductivity of composites formed by two isotropically conducting media taken in prescribed proportions. Proc. Roy. Soc. Edinburgh Sect. A 99 (1984) 71-87.

[33] M. Milgrom and M.M. Shtrickman, Linear response of two-phase composites with cross moduli: Exact universal relations. Physical Review A (Atomic, Molecular and Optical Physics) 40 (1989) 1568-1575.

[34] G.W. Milton, Bounds on the transport and optical properties of a two-component composite material, J. Appl. Phys. 52 (1981) 5294-5304.

[35] G.W. Milton, Bounds on the complex permittivity of a two-component composite material. J. Appl. Phys. 52 (1981) 5286-5293.

[36] G.W. Milton, On characterizing the set of possible effective tensors of composites: the variational method and the translation method. Comm. Pure Appl. Math. 43 (1990) 63-125.

[37] G.W. Milton, Bounds on the elastic and transport properties of two-component composites. J. Mech. Phys. Solids 30 (1982) $177-191$.

[38] G.W. Milton, The theory of composites. Cambridge Monographs on Applied and Computational Mathematics 6, Cambridge University Press, Cambridge (2002)

[39] G.W. Milton and R.V. Kohn, Variational bounds on the effective moduli of anisotropic composites. J. Mech. Phys. Solids 36 (1988) 597-629.

[40] G.W. Milton and S.K. Serkov, Bounding the current in nonlinear conducting composites. The J.R. Willis 60th anniversary volume. J. Mech. Phys. Solids 48 (2000) 1295-1324.

[41] C.B. Morrey, Multiple integral problems in the calculus of variations and related topics. Ann. Scuola Norm. Sup. Pisa 14 (1960) $1-61$

[42] F. Murat, Compacité par compensation: condition nécessaire et suffisante de continuité faible sous une hypothèse de rang constant. Ann. Scuola Norm. Sup. Pisa Cl. Sci. 8 (1982) 69-102.

[43] F. Murat and L. Tartar, Calcul des variations et homogénéisation, in Homogenization methods: theory and applications in physics (Breau-sans-Nappe, 1983), Collect. Dir. Etudes Rech. Elec. France 57, Eyrolles, Paris (1985) 319-369. English translation (see [46]).

[44] F. Murat and L. Tartar H-convergence, Séminaire d'Analyse Fonctionnelle et Numérique de l'Université d'Alger, mimeographed notes (1978). English translation (see [45]).

[45] F. Murat and L. Tartar, H-convergence, in Topics in the mathematical modelling of composite materials, Birkhauser Boston, Boston, MA, Progr. Nonlinear Differential Equations Appl. 31 (1997) 21-43

[46] F. Murat and L. Tartar, Calculus of variations and homogenization, in Topics in the mathematical modelling of composite materials, Birkhauser Boston, Boston, MA, Progr. Nonlinear Differential Equations Appl. 31 (1997) 139-173.

[47] V. Nesi, Multiphase interchange inequalities. J. Math. Phys 32 (1991) 2263-2275.

[48] V. Nesi, Bounds on the effective conductivity of 2-dimensional composites made of $n \geq 3$ isotropic phases in prescribed volume fraction: the weighted translation method. Proc. Roy. Soc. Edinburgh Sect. A 125 (1995) 1219-1239.

[49] S. Prager, Improved variational bounds on some bulk properties of a two-phase random media. J. Chem. Phys. 50 (1969) 4305-4312.

[50] C. Procesi, The invariant theory of $n \times n$ matrices. Adv. Math. 19 (1976) 306-381.

[51] E. Rogora, Invariants of matrices under the action of the special orthogonal group, preprint del Dipartimento di Matematica, Università di Roma "La Sapienza", n. 10/2005, also available at http://www.mat.uniroma1.it/people/rogora/pdf/son.pdf.

[52] K. Schulgasser, Bounds on the conductivity of statistically isotropic polycrystals. J. Phys. C10 (1977) $407-417$.

[53] S. Spagnolo, Sulla convergenza di soluzioni di equazioni paraboliche ed ellittiche. Ann. Sc. Norm. Sup. Pisa 22 (1968) 577-597.

[54] V. Šverak, New examples of quasiconvex functions. Arch. Rational Mech. Anal. 119 (1992) $293-300$.

[55] V. Šverak, Rank-one convexity does not imply quasiconvexity. Proc. Roy. Soc. Edinburgh Sect. A 120 (1992) 18-189.

[56] D.R.S. Talbot and J.R. Willis, Bounds for the effective relation of anonlinear composite. Proc. R. Soc. A 460 (2004) $2705-2723$.

[57] L. Tartar, Estimations de coefficients homogénéisés, in Computing methods in applied science and engeneering (Proc. third Int. Sympos. Versailles, 1977), Lect. Notes Math. 704, Springer Verlag, Berlin (1979) 364-373. English translation in [60].

[58] L. Tartar, Estimations fines des coefficients homogénéisés, in Ennio De Giorgi’s Colloquium (Paris 1983), P. Kree Ed., Pitman, Boston (1985) 168-187. 
[59] L. Tartar, Compensated compactness and applications to p.d.e. in nonlinear analysis and mechanics, in Heriot-Watt Symposium IV, R.J. Knops Ed., Pitman, Boston (1979) 136-212.

[60] L. Tartar, Estimations of homogenized coefficients, in Topics in the mathematical modelling of composite materials, Birkhäuser, Boston, Proc. Non Linear Diff. Equations Appl. 31 (1997) 9-20.

[61] L. Tartar, An introduction to the homogenization method in optimal design, in Optimal shape design (Tróia, 1998), Springer, Berlin, Lect. Notes Math. 1740 (2002) 47-156.

[62] L. Tonelli, Fondamenti di calcolo delle variazioni. Zanichelli, Bologna (1921).

[63] J. Von Neumann, Some matrix inequalities and metrization of metric-space Tomsk Univ. Rev. 1 (1937) 286-300 (also in Collected Works 4, 286-300).

[64] H. Weyl, The classical groups: Their invariants and representations. Fifteenth printing. Princeton Landmarks in Mathematics, Princeton Paperbacks, Princeton University Press, Princeton, NJ (1997)

[65] V.V. Zhikov, Estimates for the averaged matrix and the averaged tensor. Russian Math. Surveys 46 (1991) 65-136. 